ARTICLE

\title{
Post-endocytic sorting of Plexin-D1 controls signal transduction and development of axonal and vascular circuits
}

Katja Burk ${ }^{1, \star \dagger} \uparrow$, Erik Mire ${ }^{1, \star}$, Anaïs Bellon ${ }^{1, \star}$, Mélanie Hocine ${ }^{1}$, Jeremy Guillot ${ }^{1}$, Filipa Moraes ${ }^{2}$, Yutaka Yoshida ${ }^{3}$, Michael Simons ${ }^{2,4}$, Sophie Chauvet ${ }^{1, \star \star} \&$ Fanny Mann ${ }^{1, \star \star}$

Local endocytic events involving receptors for axon guidance cues play a central role in controlling growth cone behaviour. Yet, little is known about the fate of internalized receptors, and whether the sorting events directing them to distinct endosomal pathways control guidance decisions. Here, we show that the receptor Plexin-D1 contains a sorting motif that interacts with the adaptor protein GIPC1 to facilitate transport to recycling endosomes. This sorting process promotes colocalization of Plexin-D1 with vesicular pools of active R-ras, leading to its inactivation. In the absence of interaction with GIPC1, missorting of Plexin-D1 results in loss of signalling activity. Consequently, Gipc1 mutant mice show specific defects in axonal projections, as well as vascular structures, that rely on Plexin-D1 signalling for their development. Thus, intracellular sorting steps that occur after receptor internalization by endocytosis provide a critical level of control of cellular responses to guidance signals.

\footnotetext{
${ }^{1}$ Aix Marseille Univ, CNRS, IBDM, Marseille 13288, France. ${ }^{2}$ Yale Cardiovascular Research Center, Section of Cardiovascular Medicine, Department of Internal Medicine, Yale University School of Medicine, New Haven, Connecticut 06511, USA. ${ }^{3}$ Division of Developmental Biology, Cincinnati Children's Hospital Medical Center, Cincinnati, Ohio 45229, USA. ${ }^{4}$ Department of Cell Biology Yale University School of Medicine, New Haven, Connecticut 06511, USA. ${ }^{*}$ These authors contributed equally to this work. ${ }^{\star \star}$ These authors jointly supervised this work. †Present address: European Neuroscience Institute Göttingen (ENI-G), 37077 Göttingen, Germany. Correspondence and requests for materials should be addressed to F.M. (email: fanny.mann@univ-amu.fr).
} 
T he nervous system wires itself with remarkable precision due to the homing behaviour of axonal growth cones, whose function is dependent on membrane trafficking events. Exocytosis and endocytosis are both essential to regulate growth cone morphology and adhesive properties during axon outgrowth and guidance ${ }^{1-3}$. In particular, during chemotactic guidance, spatial asymmetry in membrane trafficking across the growth cone drives its turning response to the side with increased exocytosis, or decreased endocytosis ${ }^{4,5}$.

In addition to acting as a driving force for axon development, membrane trafficking also regulates the dynamics of cell surface receptors for extracellular ligands 6 . Endocytosis of ligandreceptor complexes from the plasma membrane has been primarily associated with desensitization of axonal responses to axon guidance cues ${ }^{7}$. However, endocytosis also critically regulates signalling from guidance cue receptors. For example, the Frizzled3 receptor requires internalization from the cell surface to activate planar cell polarity signalling during Wnt-promoted growth of spinal commissural axons ${ }^{8}$, as does the Robo receptor to recruit Son of Sevenless, a downstream effector of repulsive Slit signalling at the midline ${ }^{9}$. Shortly following endocytosis, internalized receptors are delivered to early endosomes that constitute the primary sorting station along the post-endocytic pathway. Sorting events initiated at this compartment determine the fate of internalized receptors, destining them either for recycling to the plasma membrane, transport to the Golgi or degradation in lysosomes. Potentially, signalling activity can be regulated at the level of post-endocytic sorting through spatial relocation of receptors and interaction with signalling molecules that are compartmentalized into specific endosomal vesicles ${ }^{10}$. However, little is currently known about the fate of guidance cue receptors endocytosed at the growth cone and whether post-endocytic sorting events play a role in dictating their signalling responses.

The Semaphorins define a large family of guidance cues that can elicit growth cone collapse and repulsive turning. The prototypic semaphorin, Sema3A, induces internalization of its receptor complex during repulsive axon guidance ${ }^{11}$. A recent study reported that the two Sema3A co-receptors, Neuropilin-1 and L1CAM, segregate in endosomes of different lipid composition after their co-endocytosis in growth cones of embryonic sensory neurons ${ }^{12}$. Interestingly, the adhesion molecule TAG-1 (transient axonal glycoprotein-1), which is required for Sema3A-induced collapse of sensory growth cones, has been found to facilitate endocytosis of the Neuropilin-1/ L1CAM complex and to mediate the subsequent segregation of the two proteins into different endosomal populations ${ }^{12,13}$. While this suggests a link between intracellular trafficking of co-receptor proteins and Semaphorin signalling, exactly how these two events are related to each other is unclear. Indeed, it remains to be determined how the signal-transducing elements of the Semaphorin receptor complexes, the Plexins, are trafficked inside the growth cone and whether endosomal sorting directly controls Plexin receptor activity and signal transduction.

Here, we focus on Plexin-D1, the cell surface receptor for the Semaphorin 3E (Sema3E) ligand, to investigate the interplay between post-endocytic sorting and signalling in growth cone guidance. Sema3E has the unique ability among class 3 semaphorins to bind directly to Plexin-D1 without requiring a Neuropilin as a co-receptor ${ }^{14}$. Sema3E-dependent activation of Plexin-D1 induces cell repulsion and is involved in various aspects of neuronal wiring, from axon growth and guidance to synapse formation ${ }^{15}$. Here we identify a sorting mechanism involving the PDZ domain-containing protein GIPC1 (also known as Synectin) that regulates transport of ligandactivated Plexin-D1 at trafficking checkpoints downstream of endocytosis. Interfering with this mechanism reveals that Plexin-D1 signalling in growth cones is initiated from endocytic recycling compartments and missorting of the internalized receptor causes loss of cell response to Sema3E and specific axon guidance errors in vivo. This GIPC1-dependent mechanism also regulates blood vessel guidance in vivo. Thus, we propose that the precise sorting of guidance cue receptors along the endosomal pathway provides an important level of regulation of
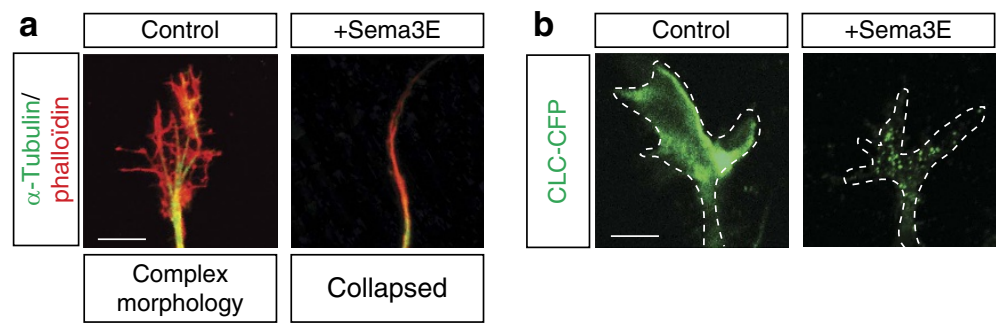

C

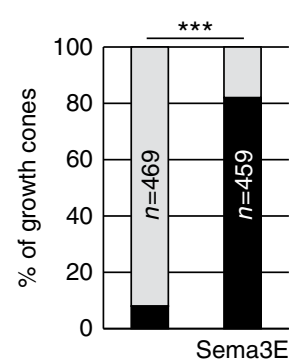

d
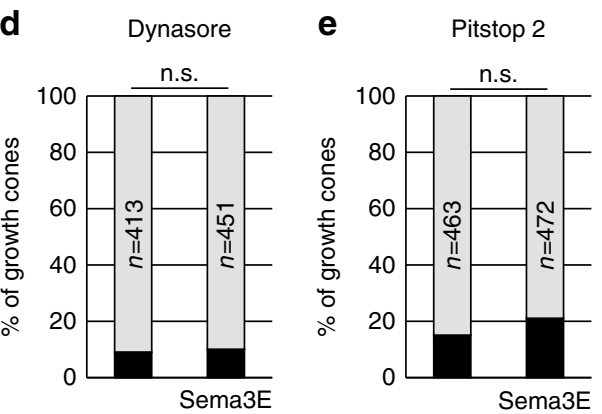

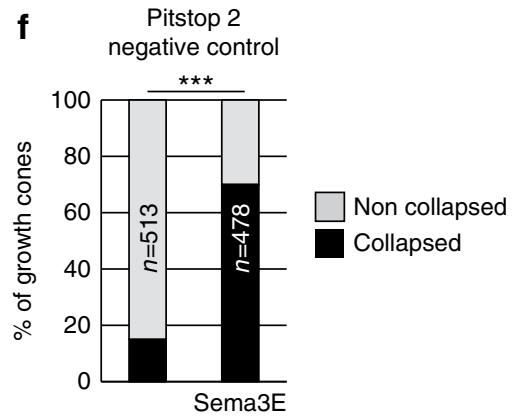

Figure 1 | Endocytosis is required for Sema3E-induced growth cone collapse. (a) Collapse assay performed on E15.5 Pir neurons identified by tubulin in the presence or absence of Sema3E (20 min of treatment). Phalloidin staining shows the complex morphology of growth cones in the control condition and the collapsed morphology in the presence of Sema3E. (b) Image of growth cones of cultured E15.5 Pir neurons expressing clathrin light chain-CFP (CLC-CFP), with or without Sema3E (10 min of treatment). (c-f) Quantification of the percentage of collapsed growth cones in control cultures and in response to Sema3E (20 min of treatment). Sema3E-induced collapse was blocked by the endocytosis inhibitors dynasore and Pitstop $2 ; n=$ number of growth cones analysed per condition in three independent experiments. The $\chi^{2}$ test, ${ }^{\star \star \star} P<0.0001$. Scale bars, $10 \mu \mathrm{m}$. See also Supplementary Fig. 1 . 

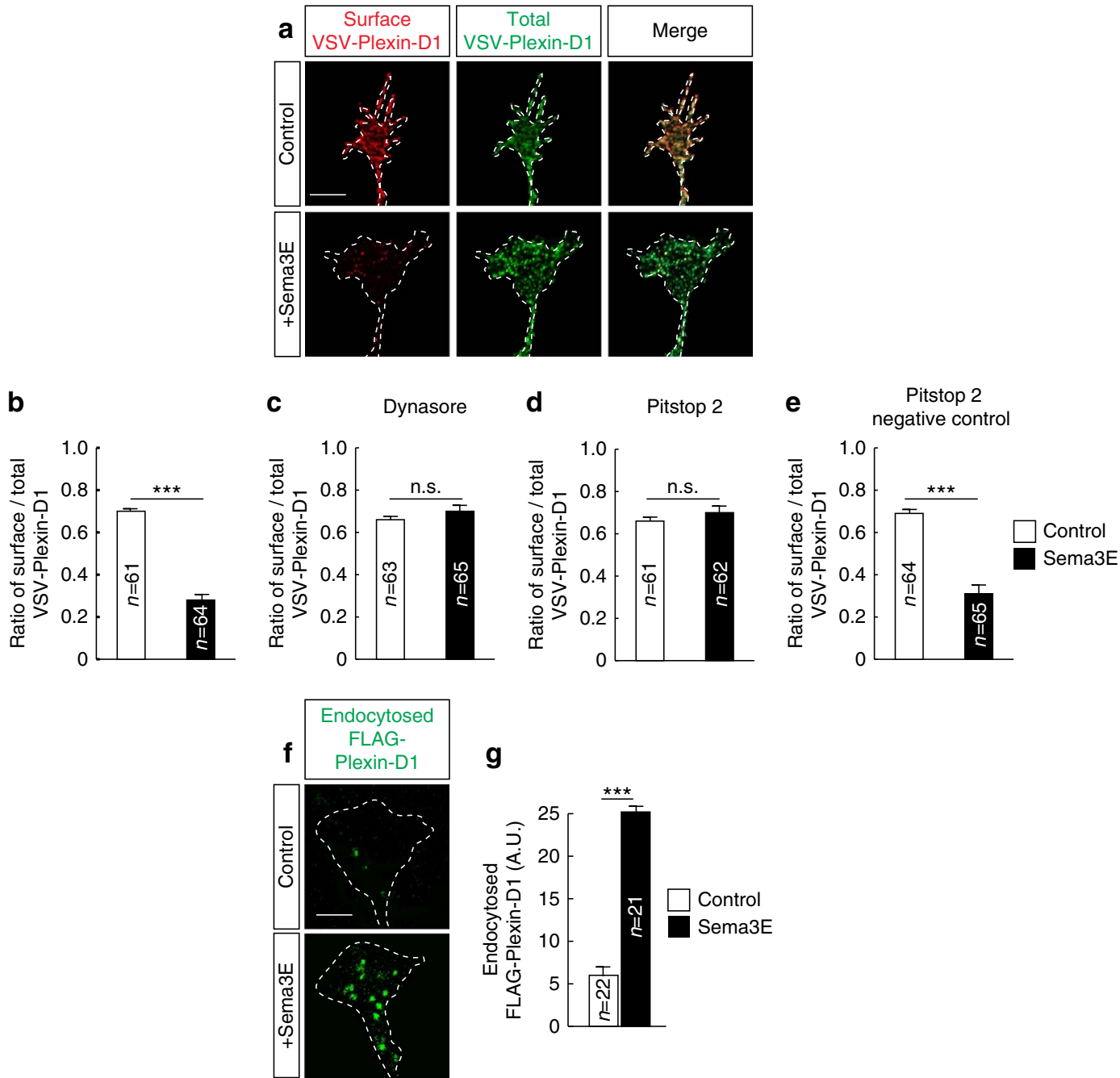

Figure 2 | Sema3E induces Plexin-D1 endocytosis. (a) Examples of growth cones from E15.5 Pir neurons showing cell surface localization (Control) and internalization ( + Sema3E) of VSV-Plexin-D1. (b-e) Quantification of the cell surface/total VSV-Plexin-D1 ratio in control growth cones and growth cones exposed to Sema3E (10 min of treatment) in the presence or absence of dynasore, Pitstop 2 or Pitstop 2-negative control. Sema3E induced clathrin- and dynamin-dependent internalization of Plexin-D1; $n=$ number of growth cones analysed per condition in three independent experiments. Data are represented as mean \pm s.e.m., ${ }^{\star \star \star} P<0.0001$ by the Mann-Whitney test. (f) Examples of growth cones from E15.5 Pir neurons showing low (Control) and high ( + Sema3E) endocytosis of FLAG-Plexin-D1. (g) Quantification of endocytosed FLAG-Plexin-D1 in growth cones illustrated in (f). Results indicate endocytosed of Plexin-D1 after Sema3E treatment; $n=$ number of growth cones analysed per condition. Data are presented as mean \pm s.e.m. and values are indicated in arbitrary units (a.u.) of fluorescence. ${ }^{\star \star} P<0.0001$ by the Mann-Whitney test. Scale bars, $10 \mu \mathrm{m}$. See also Supplementary Fig. 2.

the signalling pathways that governs the wiring of neuronal and vascular circuits.

\section{Results}

Sema3E-induced growth cone collapse requires endocytosis. Since previous studies involved endocytosis in regulating guidance receptor signalling, we sought to carefully characterize the role of endocytic trafficking in the repulsive response of growth cones to Sema3E. For this, $10 \mathrm{nM}$ Sema3E was bath-applied to Plexin-D1-expressing neurons isolated from mouse embryonic day (E) 15.5 piriform cortex (Pir) ${ }^{16}$. After $10 \mathrm{~min}$, the number of collapsed growth cones rose to $50 \%$, and reached a maximum of $\sim 85 \%$ after $20 \mathrm{~min}$ (Fig. 1a and Supplementary Fig. 1). To examine receptor-mediated endocytosis, we expressed in neurons a clathrin light chain-cyan fluorescent protein (CLC-CFP) fusion protein. A 10-min treatment with Sema3E induced the redistribution of clathrin into a punctate fluorescent pattern revealing hot spots of endocytosis that were already visible in growth cones that had not yet collapsed (Fig. 1b). We next tested the functional requirement of endocytosis for Sema3E-induced growth cone collapse by using pharmacological inhibitors of clathrin (Pitstop 2 and a negative control $)^{17}$ and dynamin (dynasore $\left.{ }^{18}\right)$. Blocking clathrin- and dynamin-dependent endocytosis completely suppressed the growth cone collapsing effect of Sema3E (Fig. 1c-f).

Sema3E promotes endocytosis of Plexin-D1 in the growth cone. We next sought to determine whether the Plexin-D1 receptor was internalized in growth cones. Although detectable, the levels of endogenous Plexin-D1 expression were too low to allow the determination of its subcellular location. Therefore, a recombinant human Plexin-D1 receptor was expressed in Pir neurons. Despite an increase of $\sim 60 \%$ in binding sites for Sema3E, Pir neurons overexpressing Plexin-D1 showed a similar 
level of collapse response to Sema3E as compared with nontransfected neurons (Supplementary Fig. 2a-c). The surface localization of exogenously expressed Plexin-D1 receptors was monitored by immunolabelling with an antibody against the extracellular domain of the human Plexin-D1 receptor, followed by cell permeabilization and labelling of the total human PlexinD1 content. After 10 min of treatment with Sema3E, the ratio of surface/total Plexin-D1 dropped from $70 \%$ in unstimulated condition to $28 \%$ (Fig. 2a,b). Pharmacological inhibitors of dynamin- and clathrin-dependent endocytosis suppressed Sema3E-induced removal of Plexin-D1 from the cell surface (Fig. 2c-e). We then confirmed endocytosis of Plexin-D1 using a live cell 'antibody feeding' assay (Fig. 2f,g) and by showing an increased colocalization of Plexin-D1 with green fluorescent
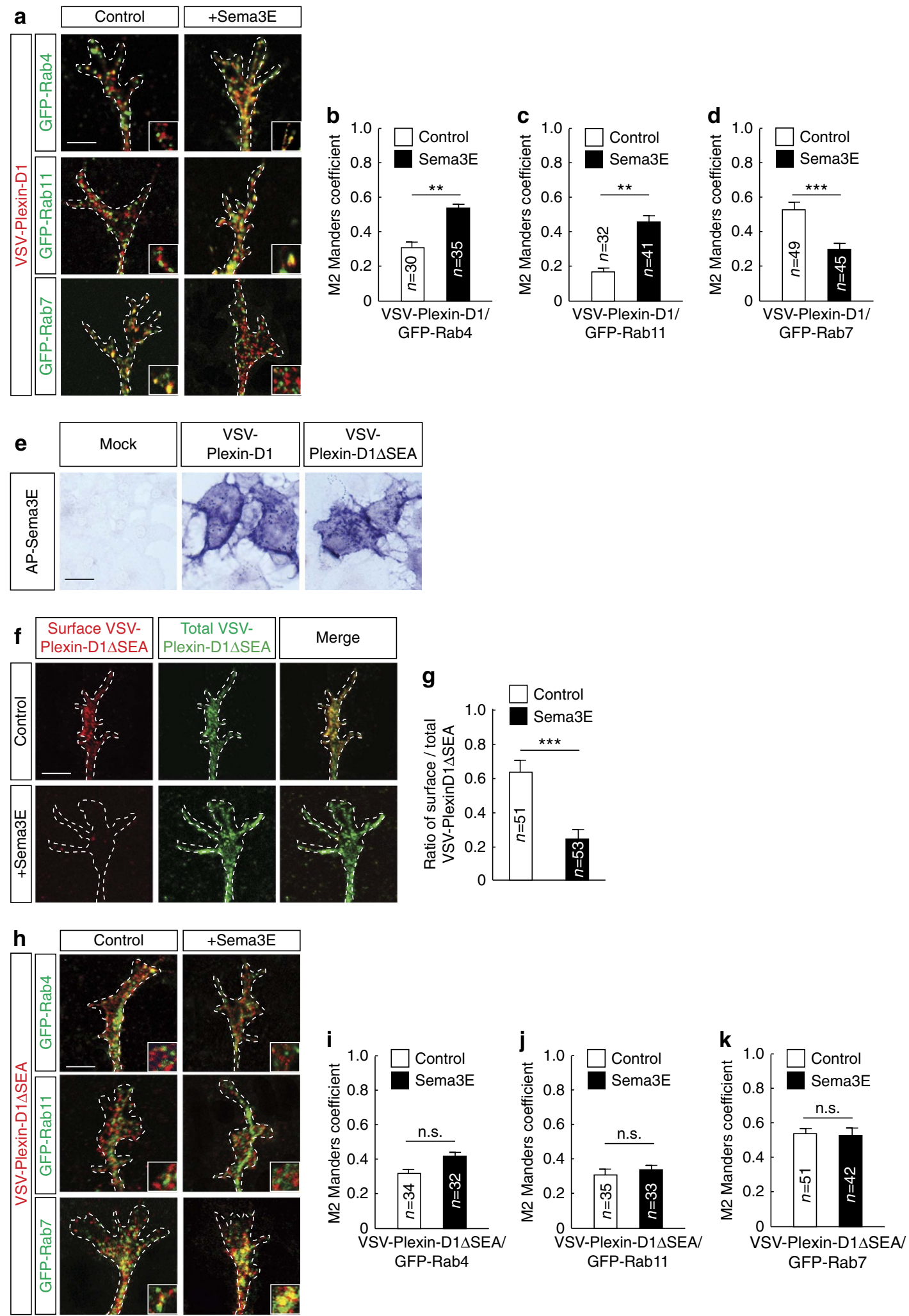
protein (GFP)-Rab5, a marker of early endosomes, in Sema3Estimulated growth cones (Supplementary Fig. 2d,e). Finally, control experiments using Sema3B and Sema3C, which do not bind directly to Plexin-D1 but induce growth cone collapse of Pir neurons, did not show endocytosis of Plexin-D1 (Supplementary Fig. 2f-h). Together, these results reveal that Plexin-D1 undergoes endocytosis in growth cones before the peak in Sema3E-induced collapse.

PDZ-dependent sorting of Plexin-D1 into recycling endosomes. We next investigated the intracellular fate of internalized Plexin-D1. In the absence of ligand, a small amount of Plexin-D1 receptors colocalized intracellularly with GFP-Rab7, a marker of late endosomes (Fig. 3a,d). In contrast, 10 min of stimulation with Sema3E enhanced the sorting of the Plexin-D1 receptor to Rab4 and Rab11 endosomes that function in rapid and slow recycling, respectively (Fig. $3 \mathrm{a}-\mathrm{c}$ ). The efficient targeting of cargo proteins to recycling endosomes often requires the presence of specific sorting motifs, such as C-terminal PDZ domain-interacting sequences ${ }^{19}$. Because Plexin-D1 harbours a class I PDZ-domainbinding motif (serine-glutamate-alanine (SEA)), we investigated the role of this sequence in receptor endocytosis and postendocytic sorting. Sema3E was able to bind to cells expressing a Plexin-D1 receptor lacking the SEA motif (Plexin-D1 $\Delta$ SEA) (Fig. $3 \mathrm{e}$ and Supplementary Fig. 2a,b) and to trigger internalization of the mutant receptor in growth cones (Fig. 3f,g), but not a collapse response (Supplementary Fig. 2c). Like the wild-type receptor, under basal conditions, the Plexin-D1 $\Delta$ SEA receptor residing intracellularly mainly distributed in late endosomes (Fig. 3h-k). However, after Sema3E stimulation, internalized wild-type and mutant receptors diverged in their post-endocytic sorting, as Plexin-D1 $\Delta$ SEA remained in GFP-Rab7 late endosomes and did not accumulate in recycling endosomes (Fig. 3h-k). Thus, binding of Sema3E relocalized the Plexin-D1 receptor from the cell surface to intracellular recycling compartments via a sorting mechanism that requires its C-terminal PDZ-binding motif.

GIPC1 controls Plexin-D1 receptor recycling. One candidate molecule that may regulate sorting of Plexin-D1 is the PDZ domain-containing protein GIPC1 that can interact with receptors containing a C-terminal SEA motif ${ }^{20}$. The interaction between Plexin-D1 and GIPC1 was confirmed in lysates of HEK293T cells coexpressing the two proteins and occurred in a ligand-independent fashion (Fig. 4a and Supplementary Fig. 4a). The SEA residues were shown to mediate this interaction, as Plexin-D1 $\Delta$ SEA did not co-precipitate with GIPC1 (Fig. 4a and Supplementary Fig. 4a). Moreover, the binding between GIPC1 and Plexin-D1 was specific among other plexins, as we found no interaction between GIPC1 and the other family members (Plexins B1, B2 and B3) harbouring a C-terminus PDZ-binding site that is structurally distinct from that of Plexin-D1 (refs 21,22) (Supplementary Figs 3a and 4b).

Gipc1 mRNA was ubiquitously expressed in the developing mouse brain (Supplementary Fig. 3b) and interaction between endogenous Plexin-D1 and GIPC1 proteins was confirmed by co-immunoprecipitation of the complex from lysate of Pir cortex (Fig. $4 \mathrm{~b}$ and Supplementary Fig. 4c). In cultured Pir neurons, GIPC1 protein was present along the length of the axons and in growth cones (Supplementary Fig. 3c,d) where it was enriched in Rab5, Rab4 and Rab11 endosomes and almost absent in Rab7 endosomes (Supplementary Fig. 3e-i). Some colocalization between Plexin-D1 and GIPC1 was observed in growing growth cones that was enhanced by stimulation with Sema3E (Fig. 4c,d), indicating that Sema3E is required to activate the plasma membrane-to-endosome traffic of Plexin-D1 and bring the two proteins in close proximity in early and/or recycling compartments of the endocytic pathway. We next determined the trafficking route of the wild-type Plexin-D1 receptor exogenously expressed in Pir neurons of Gipc1-deficient mouse embryos. GIPC1 depletion did not affect expression or surface localization of Plexin-D1 (Supplementary Fig. 3b,j-m) that was robustly internalized in growth cones within $10 \mathrm{~min}$ of application of Sema3E (Fig. 4e-h). However, the ligand-activated receptor was preferentially trafficked to the Rab7 endosomal compartment (Fig. 4i-l), similar to our observation for the mutant Plexin-D1 $\triangle$ SEA receptor. We then examined the recycling of Plexin-D1 from endosomes to the growth cone surface using a previously described assay ${ }^{23}$ (Fig. 5a). In wild-type Pir neurons, $45 \mathrm{~min}$ after stimulation with Sema3E, 63\% of internalized Plexin-D1 receptors have been recycled back to the surface of the growth cones (Fig. 5b,c). In contrast, in Gipc1-deficient neurons, the receptors were no longer recycled back to the growth cone surface (Fig. 5b,d). Furthermore, the fluorescent signal for internalized receptors had disappeared from the growth cones (Fig. 5d, no green signal in condition 3), indicating that the receptors have been degraded or transported to other location in the cell. Together, these results indicate a role for GIPC1 as an adaptor protein mediating PDZ-directed sorting of Plexin-D1 into the recycling pathway without affecting the initial step of receptor endocytosis.

GIPC1 regulates growth cone response to Sema3E repulsion. Given that GIPC1 regulates the intracellular sorting, but not internalization, of Plexin-D1, we sought to address whether modulating GIPC1 function would affect growth cone responses

\footnotetext{
Figure 3 | Sorting of Plexin-D1 into recycling pathways requires its SEA PDZ-domain-binding motif. (a) Colocalization of VSV-Plexin-D1 (red) and different GFP-tagged Rab proteins (green) in cultured E15.5 Pir neurons treated or not treated with Sema3E (10 min). (b-d) Graphs showing the Manders colocalization coefficients for the fraction of VSV-Plexin-D1 colocalized with GFP-Rab4, GFP-Rab11 or GFP-Rab7 in the presence or absence of Sema3E treatment $(10 \mathrm{~min})$. Ligand-activated Plexin-D1 receptors were directed to recycling endosomes; $n=$ number of growth cones analysed per condition in three independent experiments. Data are represented as mean \pm s.e.m., ${ }^{\star \star} P<0.01$, ${ }^{\star \star \star} P<0.0001$ by the Mann-Whitney test. (e) Alkaline phosphatase (AP)-tagged Sema3E binds equally well to COS7 cells expressing VSV-Plexin-D1 or VSV-Plexin-D1 $\triangle S E A$. No binding is observed on mock-transfected COS7 cells. (f) Examples of growth cones from E15.5 Pir neurons showing cell surface localization (Control) and internalization ( + Sema3E) of VSV-PlexinD1 $\triangle$ SEA. (g) Quantification of the cell surface/total VSV-Plexin-D1 $\triangle$ SEA ratio in control growth cones and growth cones exposed to Sema3E (10 min). Plexin-D1 lacking the SEA motif was internalized in growth cones in response to Sema3E ligand activation; $n=$ number of growth cones analysed per condition in three independent experiments. Data are represented as mean \pm s.e.m., ${ }^{\star \star \star} P<0.0001$ by the Mann-Whitney test. (h) Colocalization of VSV-Plexin-D1 $\triangle$ SEA (red) with different GFP-Rab proteins (green) in cultured E15.5 Pir neurons with or without Sema3E treatment (10 min). (i-k) Graphs showing the Manders colocalization coefficient for the fraction of VSV-Plexin-D1 $\Delta$ SEA colocalized with GFP-Rab4, GFP-Rab11 or GFP-Rab7 with or without Sema3E treatment $(10 \mathrm{~min})$. Ligand-activated Plexin-D1 $\triangle$ SEA was missorted to late endosomes; $n=$ number of growth cones analysed per condition in three independent experiments. Data are represented as mean \pm s.e.m. No statistical difference was found between conditions using the Mann-Whitney test. Scale bars, $10 \mu \mathrm{m}$. See also Supplementary Fig. 2.
} 
to Sema3E. We found that Pir neurons from $\mathrm{Gipc1}^{-/-}$mutant embryos failed to collapse upon Sema3E exposure (Fig. 6a). The collapse response was restored after the reintroduction of GIPC1 protein (Fig. 6a). Regulating growth cone repulsion was not a general function of GIPC1, however, as it was not required for the collapsing activity of Sema3B and Sema3C on Pir neurons (Fig. 6b). To test whether the interaction of GIPC1 to Plexin-D1 was directly required for the response to Sema3E, we expressed either the wild-type Plexin-D1 receptor or the mutant receptor missing the SEA motif in neurons from mouse embryos lacking endogenous Plexin-D1 (Plxnd1 $1^{l o x} /{ }^{-} ; \mathrm{Tg}$ (Nes-cre) mice; Supplementary Fig. $3 j$ ). Unlike the wild-type receptor, Plexin-D1 $\Delta$ SEA was unable to mediate Sema3E-induced collapse (Fig. 6c). Finally, we found that Gipc1 was also required for Sema3E to cause PlexinD1-dependent fasciculation of Pir axons (Fig. 6d-g). Together, these results indicate that downstream of endocytosis, the proper endosomal sorting of Plexin-D1 is required to trigger a repulsive cellular response to Sema3E. a

HEK293T cells

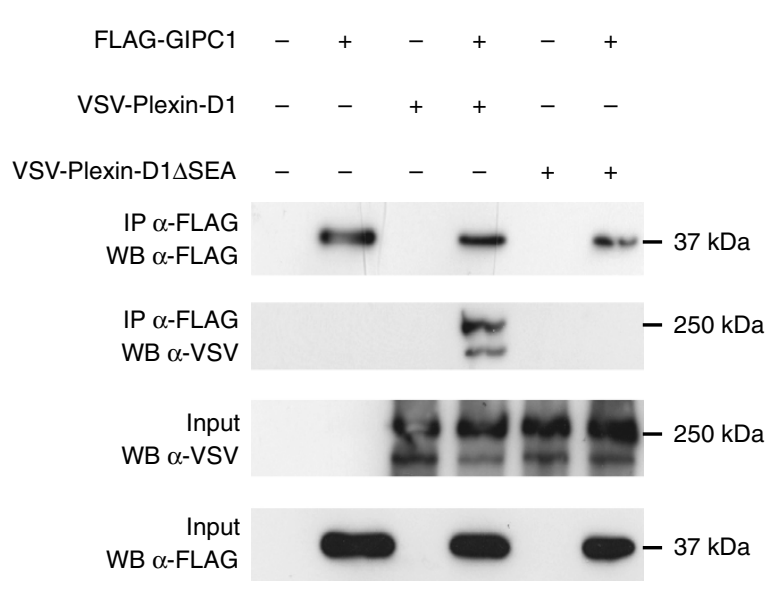

b

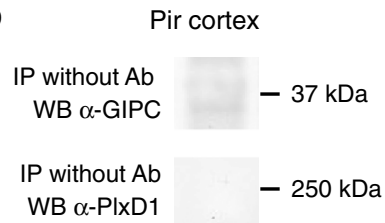

$$
\text { IP } \alpha \text {-GIPC }
$$

WB $\alpha$-GIPC

IP $\alpha$-GIPC $\quad-250 \mathrm{kDa}$
WB $\alpha$-PlexinD1

WB $\alpha$-GIPC $--37 \mathrm{kDa}$

$\underset{\mathrm{WB} \alpha \text {-PlexinD1 }}{\text { Input }}-250 \mathrm{kDa}$

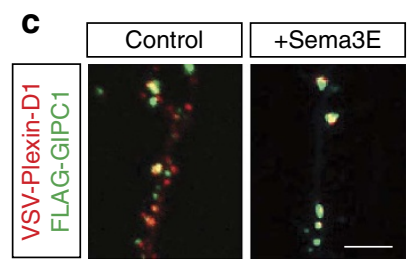

d

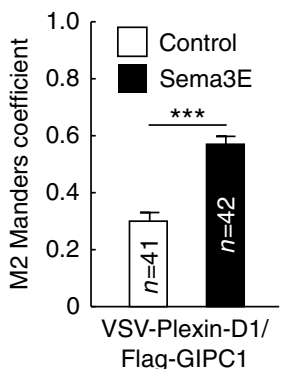

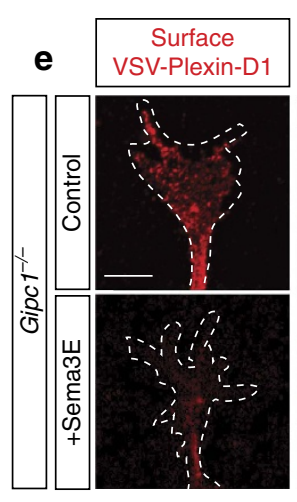
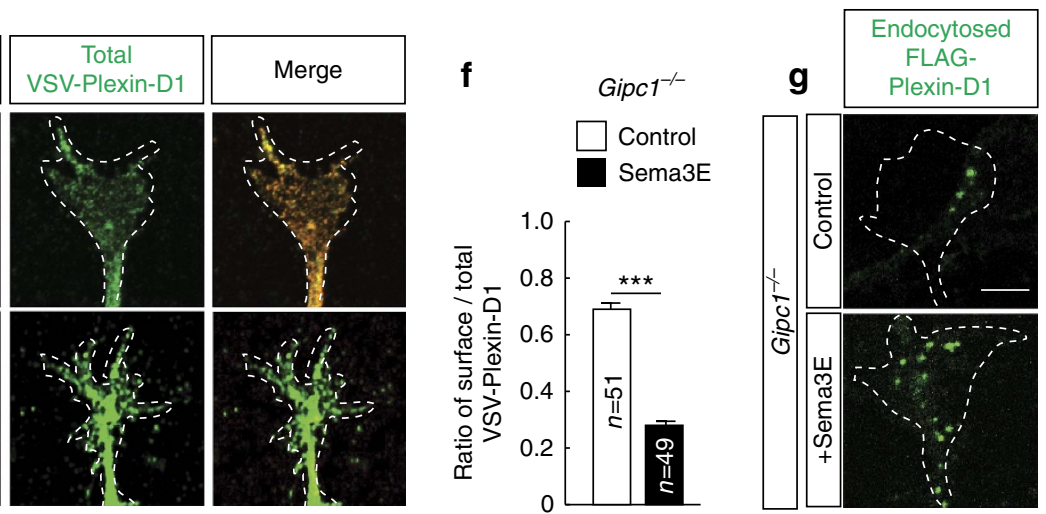

h
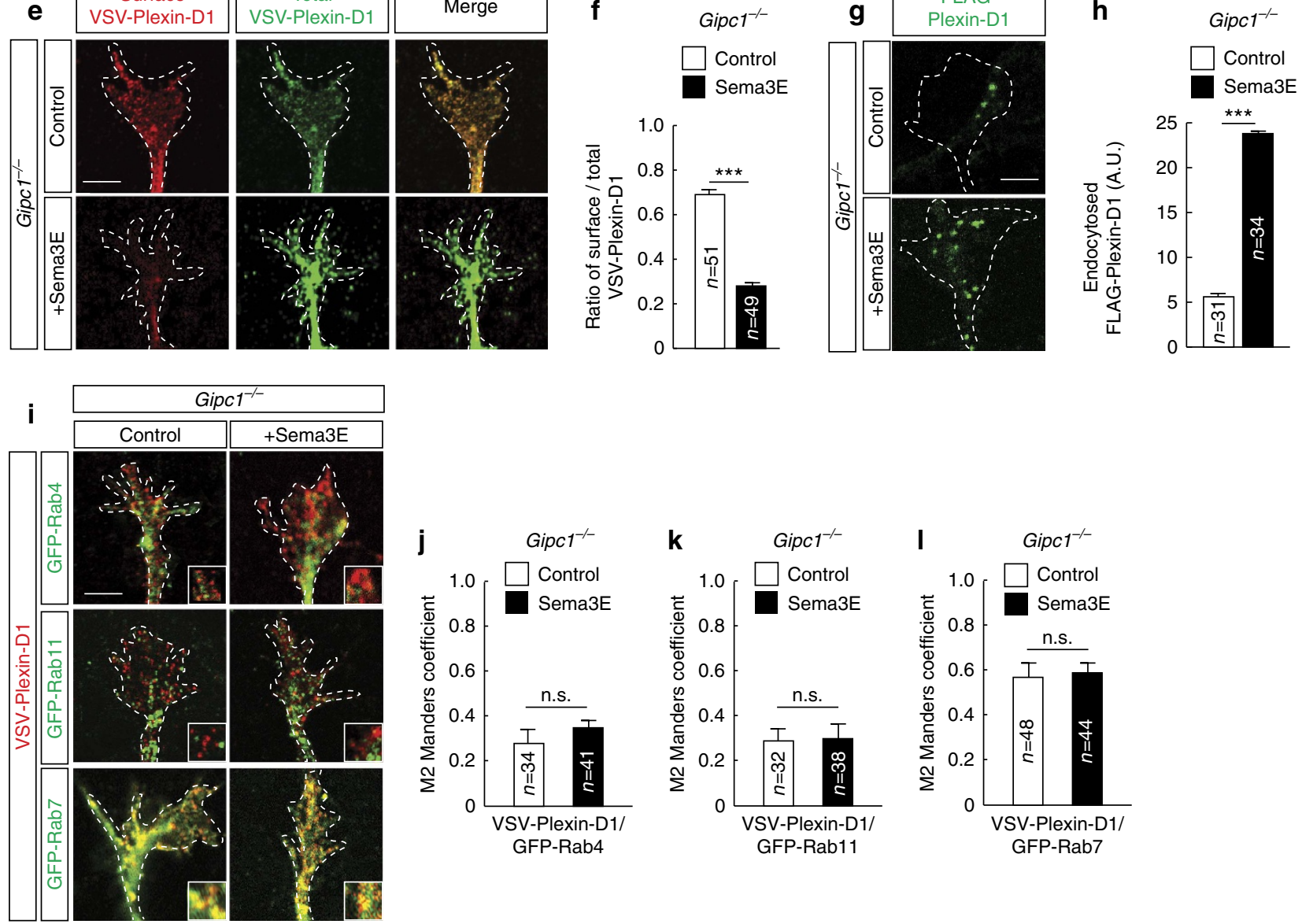
GIPC1 is required for Sema3E-induced inhibition of R-ras. Based on the above results, we hypothesized that the localization of intracellular Plexin-D1 to endosomal recycling compartments may specifically regulate signal transduction events. Previous studies reported that the activation of Plexin-D1 results in the inhibition of R-ras, a member of the superfamily of small GTPases, via its GTPase-activating protein domain ${ }^{24,25}$. Consistent with this, introducing a constitutively active R-ras $\left(\mathrm{R}-\mathrm{ras}^{38 \mathrm{~V}}\right.$ ) in Pir neurons prevented growth cone collapse induced by Sema3E (Fig. 7a). In heterologous cell lines, R-ras is enriched on vesicular structures positive for early endosomal/ recycling markers ${ }^{26}$. In growth cones, R-ras similarly distributed to Rab4- and Rab11-positive endosomes and much less in Rab7 endosomes (Supplementary Fig. 5a,b). In the absence of Sema3E ligand, little colocalization was observed between R-ras and Plexin-D1 that then constitutively traffics through Rab7 compartments (Fig. 7b,c). However, colocalization between R-ras and Plexin-D1 increased significantly after the application of Sema3E (Fig. 7b,c). Finally, little colocalization between R-ras and PlexinD1 was observed in growth cones lacking GIPC1, even after stimulation with Sema3E (Fig. 7b,d). Thus, GIPC1-dependent sorting of Plexin-D1 to specific endosomal compartments may promote a functional interaction with R-ras, a key component of the signal transduction machinery downstream of Sema3E.

To directly test whether Plexin-D1 inhibits R-ras at the level of endosomes, we used a Förster resonance energy transfer (FRET)based biosensor for R-ras, called Raichu-R-ras, that allows a direct measurement of activity change of this protein in living cells ${ }^{26}$. In Pir neurons growing on a laminin/poly-lysine substrate, R-ras activity was high in vesicular structures within axonal growth cones (Fig. 7e). This is consistent with previous studies that have implicated R-ras in mediating integrin-dependent neurite outgrowth on laminin 27,28 . Currently, however, the upstream pathway that positively regulates R-ras activity is not known. Within 3-9 min after the addition of Sema3E, the FRET signal decreased on a portion of the vesicles (40.7\%; Fig. 7e-g), indicating the inactivation of R-ras presumably in the recycling endosomes that traffic the activated Plexin-D1 receptor. In other vesicles, FRET signals increased (40.8\%) or remained unchanged (18.5\%; Fig. 7e-g). By contrast, in Gipc1 ${ }^{-1-}$ growth cones, only $12.5 \%$ of the vesicles showed decreased R-ras activity after stimulation with Sema3E, and the large majority displayed increased or unchanged FRET signals (66.7\% and 20.8\%, respectively; Fig. $7 \mathrm{~g}$ ). These data indicate that GIPC1, by bringing into close proximity ligand-activated Plexin-D1 and active R-ras, controls Sema3E-dependent inhibition of R-ras on endosomes.
We further investigated whether the reduced inactivation of R-ras in the absence of GIPC1 affects downstream signalling. R-ras is a positive regulator of the PI3K/Akt pathway ${ }^{29}$. Expression of a constitutively active form of Akt (myrAkt $\Delta 4-129)$ prevented the repulsive response to Sema3E (Fig. 7h), suggesting that Akt inhibition is required for Sema3E signalling. Indeed, we observed a marked decrease in the phosphorylation of Akt at S473 in lysates of Pir neurons stimulated for 10 min with Sema3E (Fig. 7i,j and Supplementary Fig. 6a). This process was inhibited in dynasore-treated neurons (Supplementary Fig. 5c,d) and in Gipc1 ${ }^{-1-}$ neurons (Fig. 7k,l and Supplementary Fig. 6b). Together, these data are consistent with the idea that inhibition of the R-ras/PI3K/Akt signalling cascade through the Plexin-D1 receptor is dependent on GIPC1-mediated post-endocytic sorting of the receptor into recycling pathways.

Plxnd1 and Gipc1 cooperate for axon tract formation in vivo. Our observations indicate that GIPC1-regulated sorting of PlexinD1 to recycling routes is required for receptor activity and signalling. How does this mechanism contribute to in vivo brain development? To address this question we first examined the requirement for Plxnd1 in the establishment of the anterior commissure (AC), a tract containing the axons of the Pir neurons used in the in vitro analysis. In the developing mouse brain, Plexin-D1 protein was detected on the three branches of the AC (the anterior limb, the posterior limb and the commissural component of the stria terminalis; Fig. 8a), and Plxnd1 and Gipc1 mRNA were coexpressed by neurons located in the different fields of origin of the AC that include, in addition to the Pir cortex, the anterior olfactory nucleus and the nucleus of the lateral olfactory tract $^{30,31}$ (Fig. 8b-d). Sema3e mRNA expression was detected in the globus pallidus, which is situated close to the $\mathrm{AC}$, and in cells of the bed nucleus of the stria terminalis, which surround the $\mathrm{AC}$ at the midline (Fig. 8e). This expression profile suggests a role for Sema3E/Plexin-D1 signalling in channelling AC axons together. We tested this hypothesis by analysing the development of the $\mathrm{AC}$ in mice with conditional inactivation of Plxnd1 in the nervous system (Plxnd1 ${ }^{l o x /} ; \mathrm{Tg}(\mathrm{Nes}$-cre $)$ mice) or in forebrain glutamatergic neurons with a pallial origin $\left(\right.$ Plxnd1 ${ }^{l o x /}{ }^{-} ; E m \times 1^{\text {cre }}$ mice) that include the AC neurons but not the subpallium territory through which AC axons project. The AC was labelled with an anti-L1CAM antibody on coronal and sagittal sections of E17.5 brains (Fig. 9a-d). In both genotypes, the AC appeared enlarged in regions close to the brain midline (Fig. $9 \mathrm{c}-\mathrm{f}, \mathrm{j}, \mathrm{k}$ ), despite normal brain size (Supplementary Fig. 7a). This enlargement was observed from E14.5, when the first commissural axons crossed the

Figure 4 | GIPC1 controls post-endocytic sorting of Plexin-D1. (a) HEK293T cells were transfected with FLAG-GIPC1, VSV-Plexin-D1 and VSV-PlexinD1 $\triangle$ SEA constructs. Proteins were immunoprecipitated (IP) from cell lysates and immunoblotted (WB) using the indicated antibodies. The C-terminal SEA motif of Plexin-D1 interacts with GIPC1. (b) Co-IP of endogenous GIPC1 and Plexin-D1 proteins from cell lysate of E15.5 Pir cortex. (c) Axons of E15.5 Pir neurons expressing FLAG-GIPC1 (green) and VSV-Plexin-D1 (red), with or without Sema3E treatment (10 min). (d) Graph showing the Manders colocalization coefficients for the fraction of VSV-Plexin-D1 colocalized with FLAG-GIPC1. Sema3E increased the colocalization of the two proteins; $n=$ number of growth cones analysed per condition in three independent experiments. Data are represented as mean \pm s.e.m., $\star \star \star ~ P<0.001$ by the MannWhitney test. (e) Growth cones of E15.5 Gipc1-/- Pir neurons showing cell surface localization (Control) and internalization ( + Sema3E) of VSV-PlexinD1. (f) Quantification of the cell surface/total VSV-Plexin-D1 ratio in Gipc1-/- growth cones. Sema3E induced internalization of Plexin-D1; $n=$ number of growth cones analysed per condition in three independent experiments. Data are represented as mean \pm s.e.m., ${ }^{\star \star \star} P<0.0001$ by the Mann-Whitney test. (g) Examples of growth cones of E15.5 Gipc1-/- Pir neurons showing low (Control) and high ( + Sema3E) endocytosis of FLAG-Plexin-D1. (h) Quantification of endocytosed FLAG-Plexin-D1 in Gipc1-/- growth cones. Sema3E induced internalization of Plexin-D1; $n=$ number of growth cones analysed per condition. Data are presented as mean \pm s.e.m. and values are indicated in arbitrary units (A.U.) of fluorescence. ${ }^{\star \star \star} P<0.0001$ by the Mann-Whitney test. (i) Colocalization of VSV-Plexin-D1 (red) with different GFP-Rab proteins (green) in E15.5 Gipc1 ${ }^{-/-}$Pir neurons with or without Sema3E treatment (10 $\mathrm{min})$. (j-I) Graphs show the Manders colocalization coefficients for the fraction of VSV-Plexin-D1 colocalized with GFP-Rab proteins in $\mathrm{Gipc1}^{-/-}$growth cones. Ligand-activated Plexin-D1 was missorted to late endosomes; $n=$ number of growth cones analysed per condition in three independent experiments. Data are represented as mean \pm s.e.m. No statistical difference was found between conditions using the Mann-Whitney test. Scale bars, $10 \mu \mathrm{m}$. See also Supplementary Figs 3 and 4. 
a

Red = Surface FLAG-Plexin-D1 labelled

by a secondary antibody

under non-permeabilizing condition

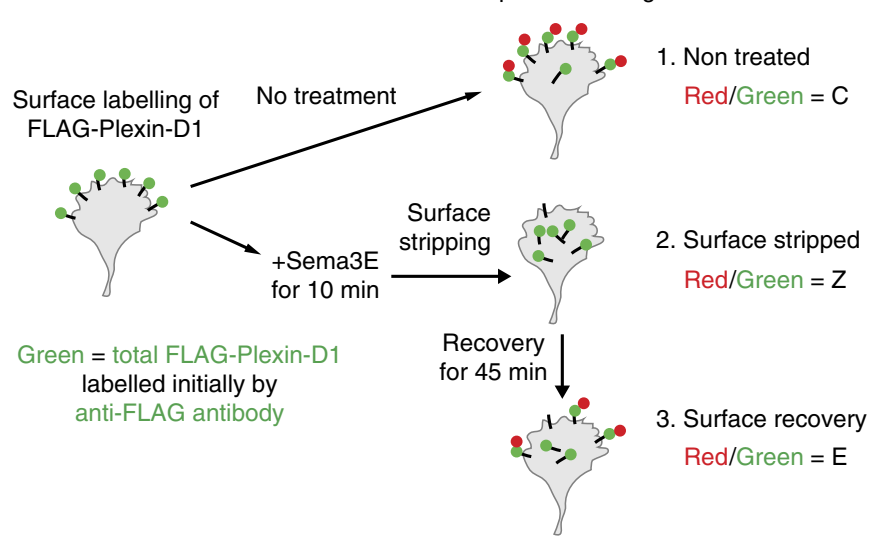

b

$\%$ of recycling $=(E-Z) /(C-Z) \times 100$
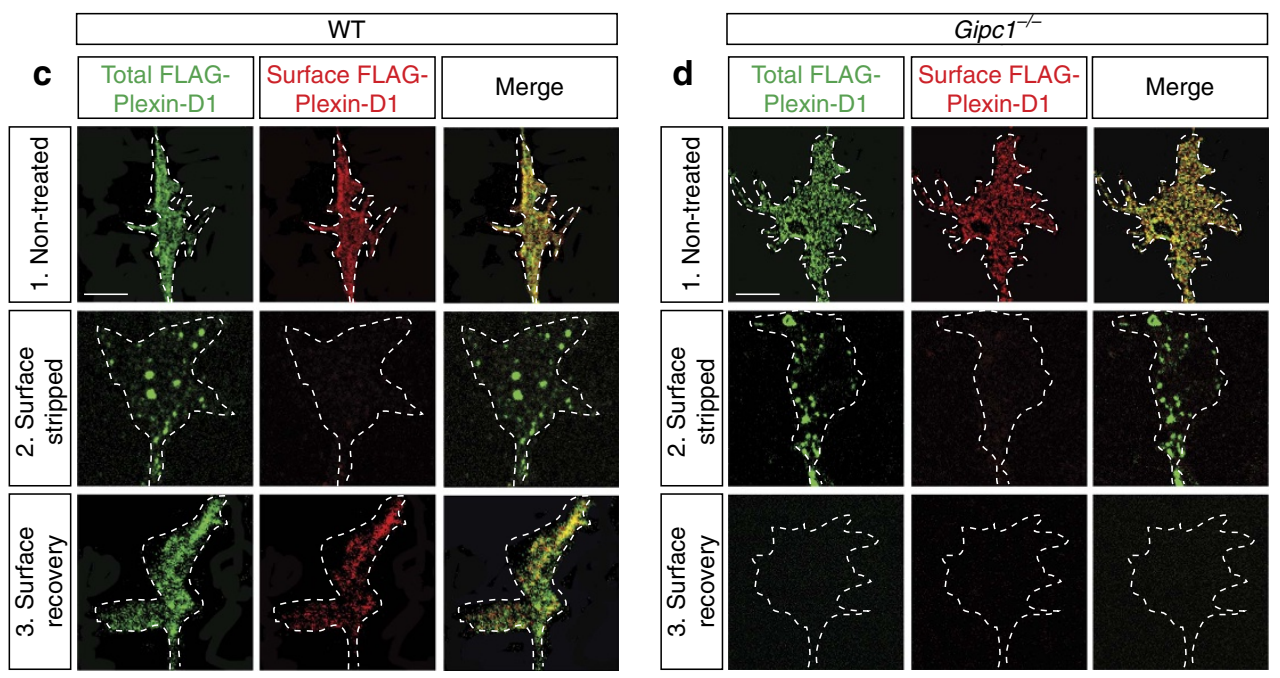

Figure 5 | GIPC1 controls Plexin-D1 receptor recycling to the plasma membrane. (a) Schematic of the quantitative receptor recycling assay. (b) Quantification of the percentage of FLAG-Plexin-D1 recycling to the growth cone surface of wild-type (WT) or Gipc1-/- E15.5 Pir neurons. GIPC1 is required for recycling of internalized Plexin-D1 to the plasma membrane; $n=$ number of growth cones analysed per condition. Data are represented as mean \pm s.e.m., ${ }^{\star \star \star} P<0.0001$ by the Mann-Whitney test. (c,d) Representative confocal fluorescence images of FLAG-Plexin-D1 recycling assay in growth cones of WT (c) and $\mathrm{Gipc}^{-/-}$(d) E15.5 Pir neurons. Scale bars, $10 \mu \mathrm{m}$.

brain midline, and persisted at least until postnatal day $(\mathrm{P})$ 30, after the development of the AC has finished (Supplementary Fig. $7 \mathrm{~b}, \mathrm{c})$. We verified that the number of projection neurons in the Pir, anterior olfactory nucleus and nucleus of the lateral olfactory tract did not vary in Plxnd1 ${ }^{l o x /}{ }^{-} ; \mathrm{Tg}$ (Nes-cre) embryos compared with controls (Supplementary Fig. $7 \mathrm{~d}-\mathrm{g}$ ), indicating that Plxnd1 deletion did not affect the generation and specification of neurons. In some contexts, AC hyperplasia might serve as a compensatory mechanism for the congenital absence of another cortical commissure, the corpus callosum ${ }^{31-33}$. However, no sign of corpus callosum dysgenesis or misrouting of neocortical axons towards the AC was found in Plxnd1 lox/-;Tg(Nes-cre) embryos (Supplementary Fig. 7h,i). Together, these data indicate that Plexin-D1 acts cell autonomously to regulate the development of the AC.

We next asked whether GIPC1 might contribute to Plexin-D1 function in this system. In E17.5 embryos with constitutive $\left(\mathrm{Gipc1}^{-/-}\right)$or conditional deletion of Gipc1 in neurons of the AC $\left(G i p c 1^{l o x}{ }^{-} ; E m x 1^{c r e}\right)$, the AC was larger than in control embryos (Fig. 9c,d,g-k; Supplementary Fig. 7a). Last, animals harbouring double heterozygous mutations for Plxnd1 and Gipc1 also displayed a significant increase in AC size that was not observed in either single Plxnd $1^{-/+}$or Gipc1 ${ }^{-/+}$heterozygous mutants (Fig. 9c,d,i-k; Supplementary Fig. 7a). Together, these data demonstrate that GIPC1 together with Plexin-D1 play a critical role in the formation of a major axon tract from the cerebral cortex.

To further explore how general is the requirement for GIPC1 in the development of Plexin-D1-expressing axonal projections, we performed additional characterization of the Plxnd1 and Gipc1 mutants and compared the results against known phenotypes of Sema3e gene alterations. Previous studies identified a role for Sema3E expression in the globus pallidus and reticular thalamic nucleus in the development of the striatonigral pathway 16,34 . Labelling of striatal projections with an antiDARPP-32 (Dopamine- and cAMP-regulated neuronal phosphoprotein 32) antibody in brains of adult Plxnd1 lox/ ${ }^{-} ;$Tg(Nes-cre), Gipc1 $1^{-1-}$ and double Plxnd1 ${ }^{-1+} ; G_{i p c 1}-1+$ heterozygous mutant mice revealed in each mutant genotype an enlargement of the striatonigral tract (Fig. 10a-c). Altogether, these data demonstrate in two distinct populations of neurons that Plexin-D1 and GIPC1 interact in the same molecular pathway to properly control axon projection patterns. 
a
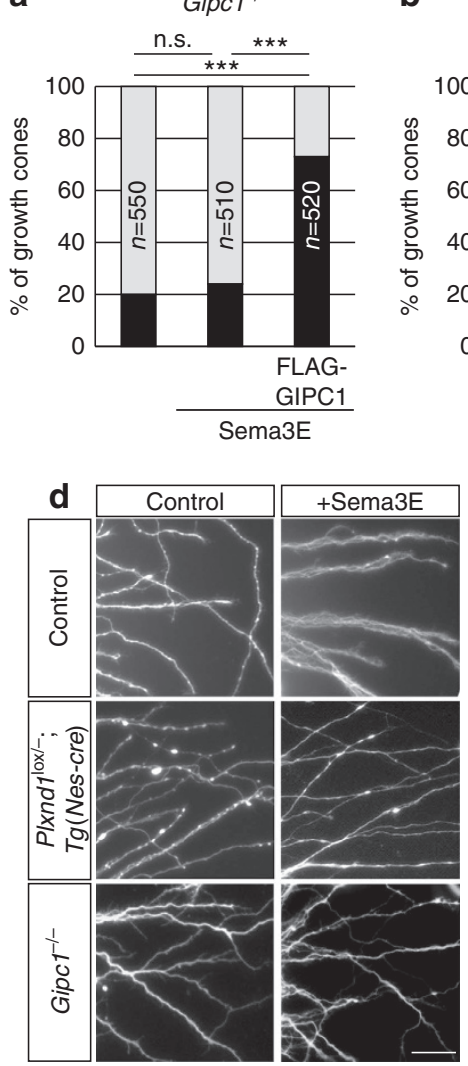

b

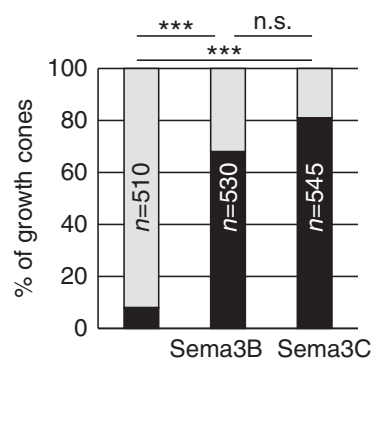

C
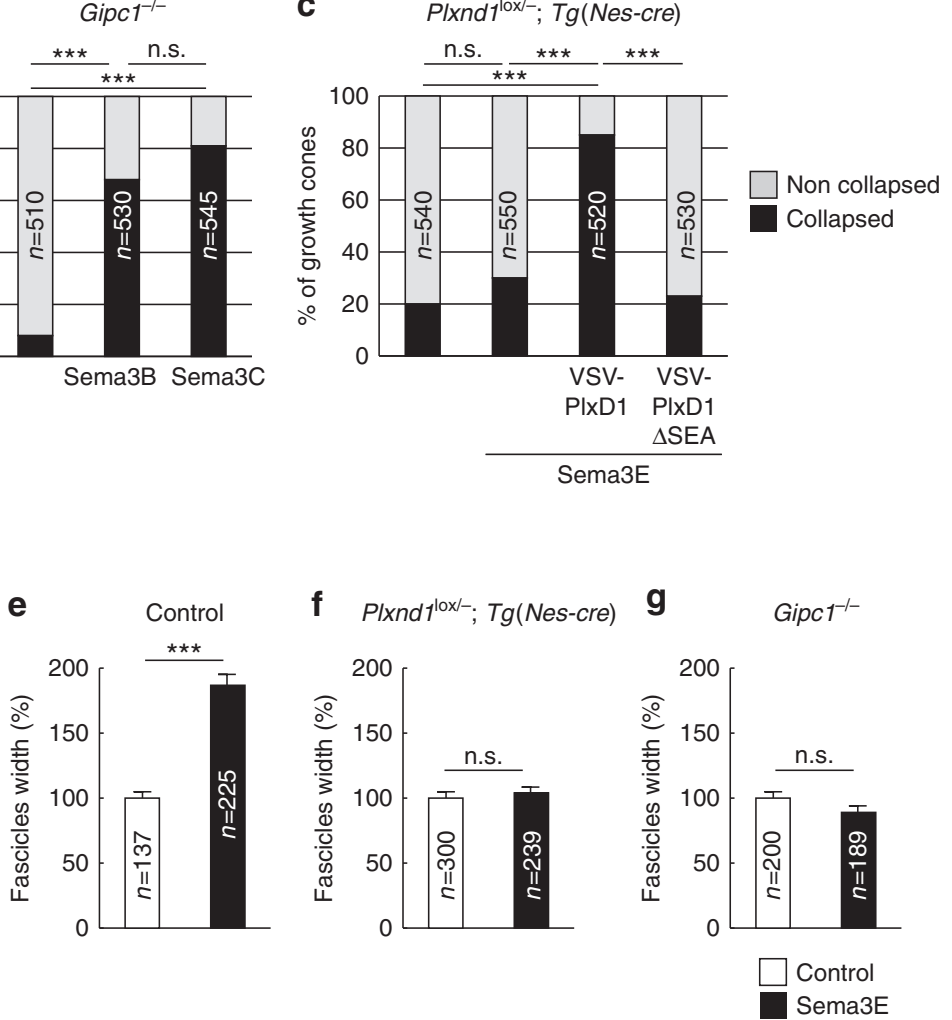

Figure 6 | GIPC1 is required for axonal and growth cone response to Sema3E. (a-c) Quantification of the percentage of collapsed growth cones in response to 20 min of treatment with Sema3E, Sema3B or Sema3C in cultures of E15.5 Pir neurons of Gipc1 ${ }^{-/-}$or Plxnd7lox/-; Tg(Nes-cre) mutants. Sema3E-induced collapse required functional GIPC1 and the C-terminal SEA motif of Plexin-D1; $n=$ number of growth cones analysed per condition in three independent experiments. The $\chi^{2}$ test, ${ }^{\star \star \star} P<0.001$. (d) Photomicrographs showing axons stained with calcein-AM growing out from E15.5 Pir explants of control, Plxnd7 lox/- $\operatorname{Tg}$ (Nes-cre) or Gipc1-/- mutants, cultured for 2 days with or without Sema3E. (e-g) Quantification of the average fascicle width in response to Sema3E in cultures of control, Plxnd7 lox $/-; \mathrm{Tg}$ (Nes-cre) or Gipc1 ${ }^{-/-}$mutant explants. Sema3E-induced fasciculation required expression of Plexin-D1 and GIPC1 in axons; $n=$ number of fascicles measured per condition in three independent experiments. Data are shown as mean \pm s.e.m. and are normalized to the values obtained in unstimulated conditions. ${ }^{\star \star \star} P<0.001$, by the Mann-Whitney test. Scale bar, $50 \mu \mathrm{m}$.

Sema3E promotes axon growth independently of GIPC1. In addition to its repulsive activity, Sema3E can also attract and promote the growth of efferent axons of the subiculum ${ }^{16}$. In E17.5 mouse embryos lacking either Sema3e or Plxnd1, very few axons reached the postcommissural part of the fornix tract ${ }^{16}$. In this particular context, Plexin-D1 is required on axons for Sema3E ligand binding but not for signal transduction that is initiated by the co-receptor vascular endothelial growth factor receptor-2 (VEGFR-2) ${ }^{35}$. If Plexin-D1 does not directly convey signal, then Gipc1 loss of function would not be expected to affect fornix development. Indeed, we found that the postcommissural fornix was formed normally in Gipc1 null mutants and in double heterozygous mutants for Plxnd1 and Gipc1 (Supplementary Fig. 8a-d). This independence for GIPC1 function was confirmed in vitro, as shown by the ability of Sema3E to stimulate elongation of subicular axons lacking Gipc1 or expressing the PlexinD1 $\triangle$ SEA mutant receptor (Supplementary Fig. 8e-g). Thus, GIPC1 is specifically required during brain development for controlling Sema3E-dependent axonal repulsion, but not elongation.

Plxnd1 and Gipc1 cooperate during vascular patterning. Finally, we speculated that the GIPC1-dependent regulation of the post-endocytic sorting and signalling of the Plexin-D1 receptor might be a general mechanism that operates in other cell types outside the nervous system. In the trunk region of mouse embryos, expression of Sema3e in somites repels the growth of adjacent intersomitic blood vessels (ISVs) that express Plexin-D1 (ref. 14). Here we found that in E11.5 Gipc1 ${ }^{-/}$embryos, ISVs labelled with anti-PECAM-1 (platelet-endothelial cell adhesion molecule-1) antibody ectopically extended throughout the somites, resulting in a loss of their normal segmental organization (Fig. 10d,e). This phenotype was similar to that reported in mice lacking Plxnd1 (ref. 14). Moreover, double Plxnd $1^{+/-}$, Gipc1 $1^{+/-}$heterozygous mutants showed a similar disturbed pattern of ISV organization (Fig. 10e). These data support an extended role for the adaptor protein GIPC1 in controlling repulsive Sema3E/Plexin-D1 signalling during patterning of the developing mouse vasculature.

\section{Discussion}

This study uncovers a specific role for the adaptor protein GIPC1 in coupling endosomal sorting of the Plexin-D1 receptor to the initiation of repulsive guidance signalling. Because ligand-induced internalization has been reported for several members of the plexin family ${ }^{11,12,36}$, our results raise the question of how general the regulation of plexin signalling by active intracellular trafficking may be. The cytoplasmic Ras GAP domains are 

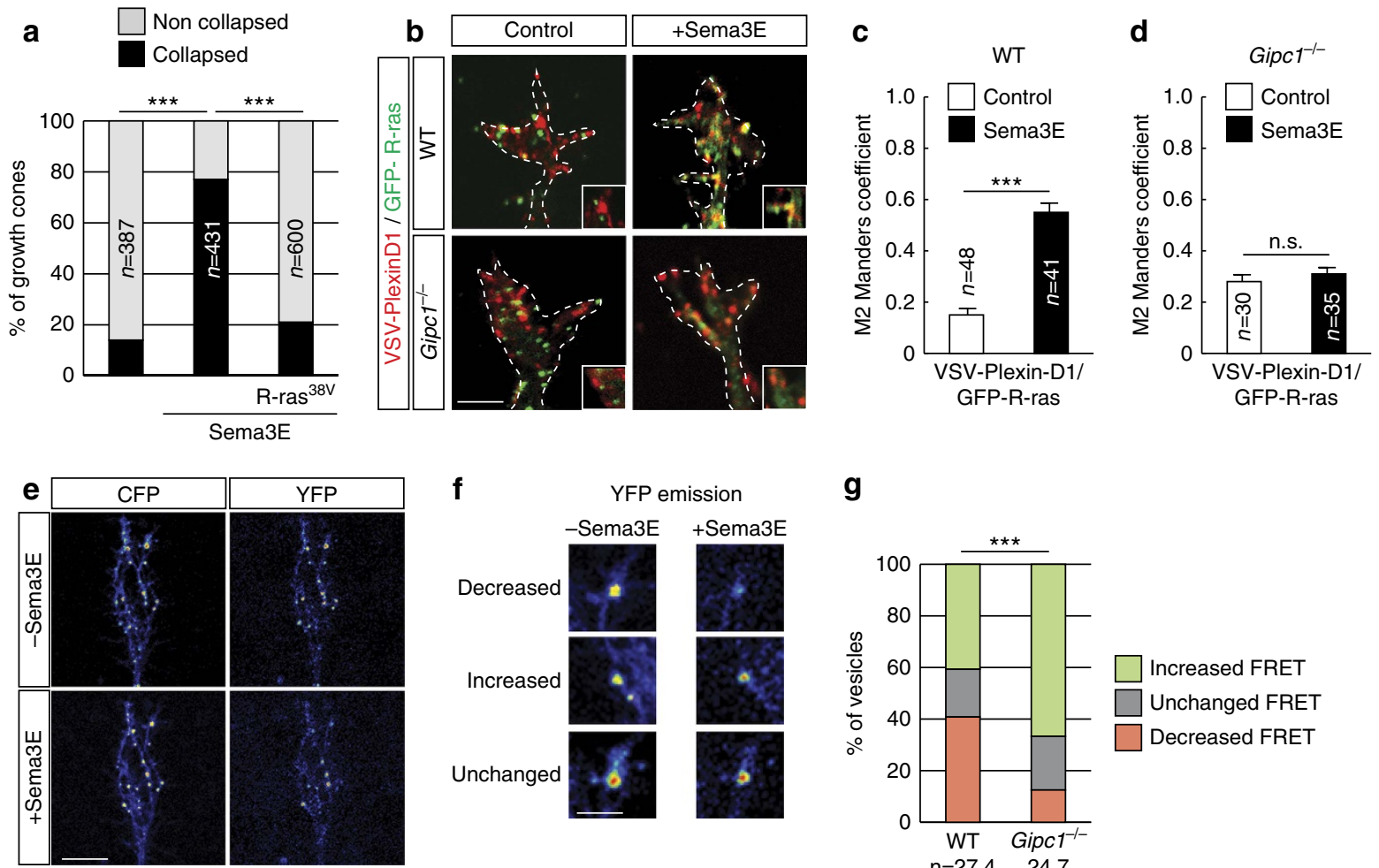

g
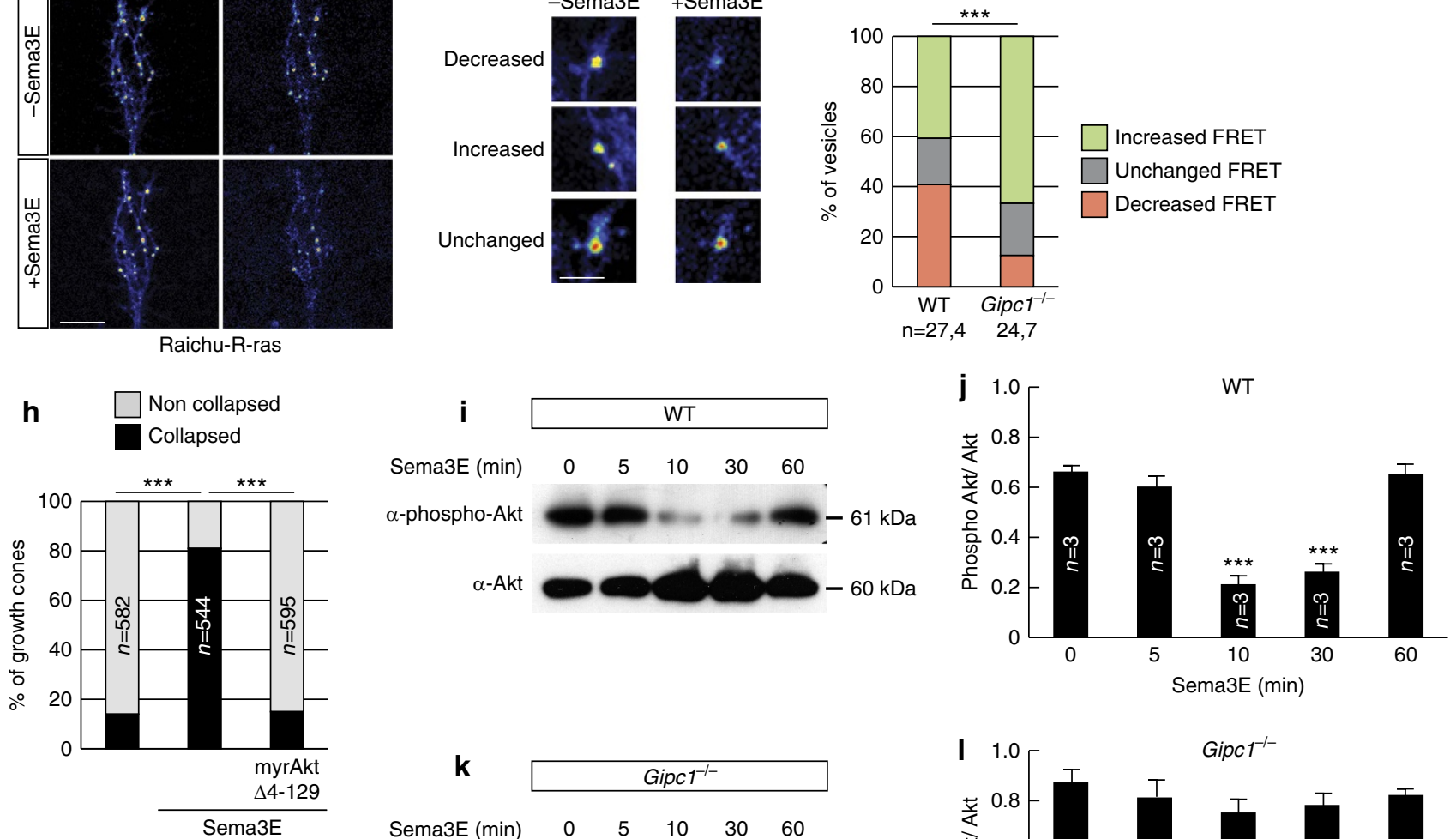

$\mathbf{k}$

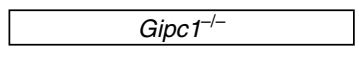

Sema3E (min)

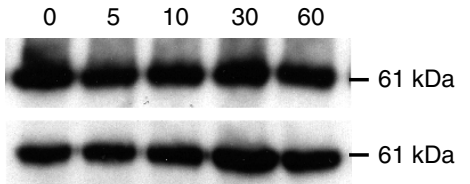

$\alpha-A k t$

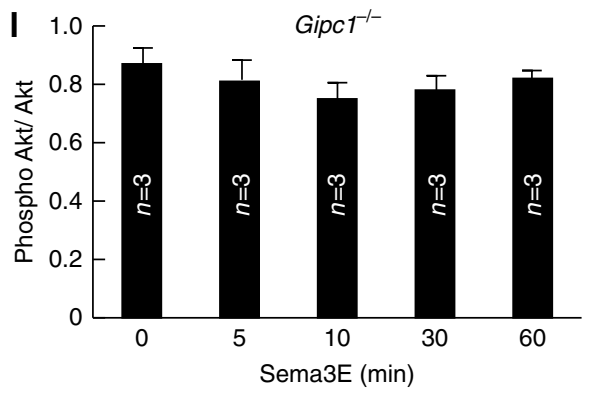

Figure 7 | Impaired signal transduction in neurons lacking GIPC1. (a) Percentage of collapsed growth cones of E15.5 Pir neurons in response to Sema3E (20 min). The constitutively active form of R-ras (R-ras ${ }^{38 \mathrm{~V}}$ ) abrogated the collapsing effect of Sema3E; $n=$ number of growth cones per condition in three independent experiments. The $\chi^{2}$ test, ${ }^{\star \star \star} P<0.0001$. (b) Growth cones of E15.5 wild-type (WT) or Gipc1-/- Pir neurons expressing GFP-R-ras and VSV-Plexin-D1, treated with or without Sema3E (10 min). (c,d) Graphs show Manders colocalization coefficients for the fraction of VSV-Plexin-D1 colocalized with GFP-R-ras. GIPC1 increased colocalization of Plexin-D1 and R-ras; $n=$ number of growth cones per condition in three independent experiments. Data are represented as mean \pm s.e.m., ${ }^{\star \star}{ }^{*} P<0.001$ by the Mann-Whitney test. (e) Expression of the Raichu-R-ras reporter in a E15.5 Pir neuron before and after the addition of Sema3E. CFP and YFP images are presented as pseudocolour images (red: high signal, blue: low signal). The CFP image (left) shows the distribution of R-ras on vesicles. The YFP signal (right) is proportional to the amount of GTP bound to R-ras. (f) Examples of changes in the YFP signal induced by exposure to Sema3E. (g) Percentage of vesicles displaying increased, decreased or unchanged FRET level. Sema3Edriven R-ras inhibition was reduced in Gipc $1^{-/-}$neurons; $n=x, y$ where $x$ indicates the number of vesicles and $y$ the number of growth cones analysed. The $\chi^{2}$ test, ${ }^{\star \star \star} P<0.0001$. (h) Percentage of collapsed growth cones in response to Sema3E (20 min) in cultures of E15.5 Pir neurons. A constitutively active form of Akt (myrAkt $\Delta 4-129$ ) abrogated the collapsing effect of Sema3E; $n=$ number of growth cones per condition in three independent experiments. The $\chi^{2}$ test, ${ }^{\star \star \star} P<0.0001$. (i,k) Phosphorylation of Akt in E15.5 WT or Gipc1-/- Pir neurons stimulated with Sema3E (0 to 60 min). (j,I) Quantification of phospho-Akt levels. Sema3E-induced inhibition of Akt required GIPC1 function; $n=$ number of experiments, data are mean \pm s.e.m., $\star \star \star P<0.001$ by the Mann-Whitney test. Scale bars, $10 \mu \mathrm{m}$ (b,e), $2 \mu \mathrm{m}$ (f). See also Supplementary Figs 5 and 6. 

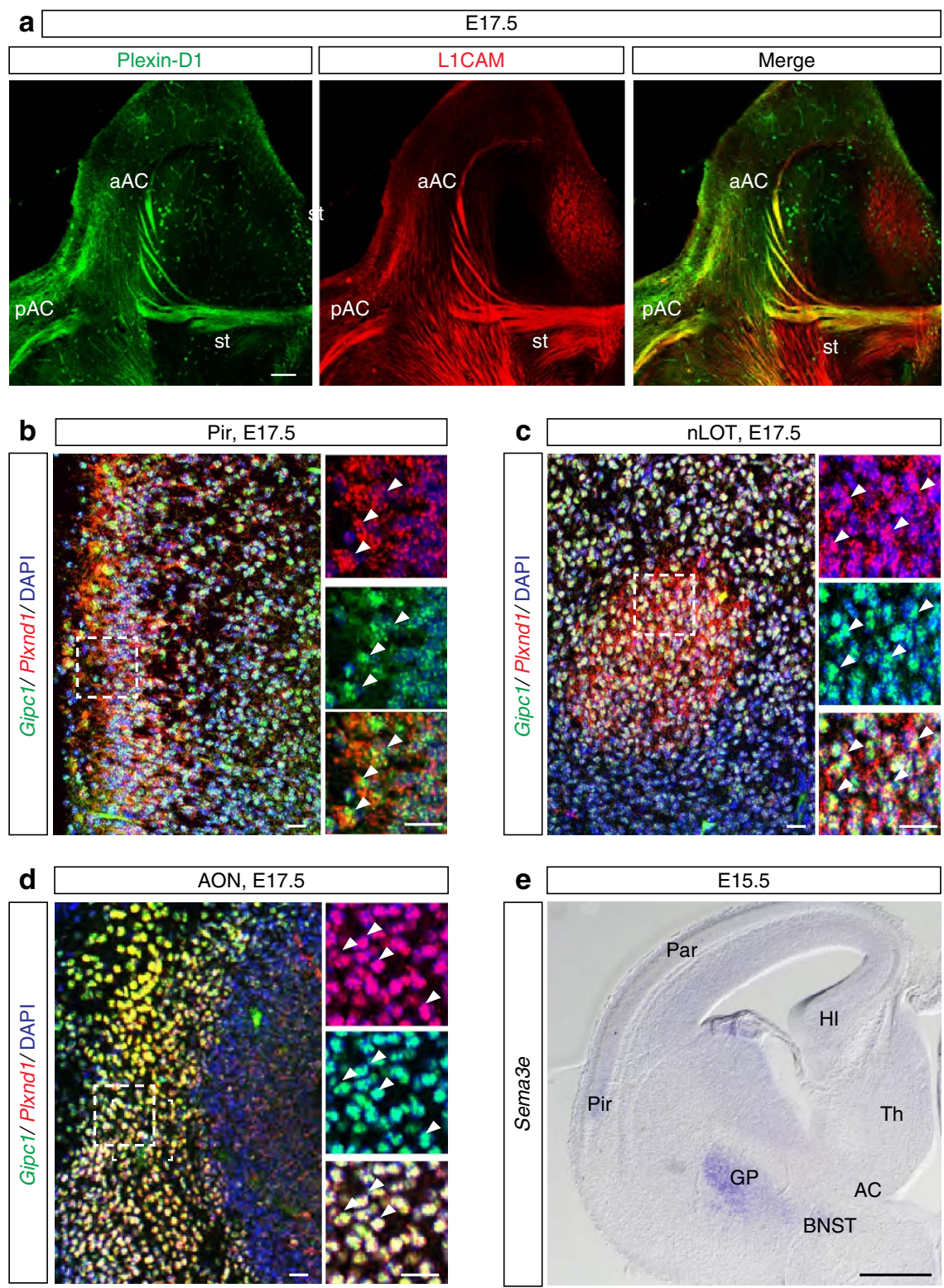

Figure 8 | Plexin-D1 and GIPC1 are coexpressed in neurons of the AC. (a) Immunolabelling of Plexin-D1 (green) and L1CAM (red) in horizontal sections of E17.5 brain. Plexin-D1 is expressed on the three branches of the AC. (b-d) Fluorescent RNA in situ hybridization for Gipc1 (green) and Plxnd1 (red) on coronal sections of E17.5 wild-type mouse brain. Gipc1 and Plxnd1 mRNA are coexpressed in the Pir cortex (b), nLOT (c) and AON (d). (e) Coronal sections of E15.5 wild-type mouse brains were hybridized with an RNA probe for Sema3e. Strong signal was seen in the GP and BNST. aAC, anterior limb of the AC; $A O N$, anterior olfactory nucleus; BNST, bed nucleus of the stria terminalis; GP, globus pallidus; HI, hippocampus; nLOT, nucleus of the lateral olfactory tract; pAC, posterior limb of the AC; Par, parietal cortex; Pir, piriform cortex; st, stria terminalis; Th, thalamus. Scale bars, $300 \mu \mathrm{m}(\mathbf{a}), 50 \mu \mathrm{m}(\mathbf{e})$ and $20 \mu \mathrm{m}$ (b-d).

highly conserved among all plexin subfamilies and, so far, three small GTPases of the Ras family, R-ras, M-ras and Rap1, have been identified as targets of plexin activity ${ }^{37-39}$. Although it remains controversial which of these Ras family proteins is the most relevant for semaphorin-mediated repulsion, they have all been shown to reside primarily on endosomes. However, they exhibit different subcellular localizations: whereas activated R-ras signals at the membrane of recycling endosomes, Rap1 activity is mainly associated with late endosomes ${ }^{40,41}$. Thus, an attractive hypothesis is that receptor endocytosis is a general mechanism by which plexins inhibit the activation of Ras proteins on endosomes and that some specificity towards the different Ras protein isoforms may be achieved through spatial restriction by postendocytic sorting into distinct intracellular trafficking routes.
The question of how the Plexin-D1 receptor uses GIPC1 to enter the recycling pathways remains unanswered. One current model proposes that recycling depends on the capacity of internalized receptors to enter into specialized tubular microdomains of the early endosomes that undergo scission and transport to the cell surface via direct or slow indirect routes. A crucial element of the machinery that recruits cargo receptors into these endosomal tubules is a pentameric protein complex termed 'retromer'42. Interestingly, a recent proteomic study of non-neuronal cells identified several plexins, including Plexin-D1, as cargo proteins of the retromer ${ }^{43}$. The retromer constitutes a central platform for the recruitment of a number of accessory proteins that aid in cargo sorting. Among these, the WASH (Wiskott-Aldrich syndrome protein and SCAR homologue) 
a

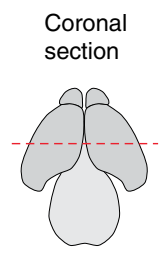

E17.5
Labelling by anti-L1CAM antibody

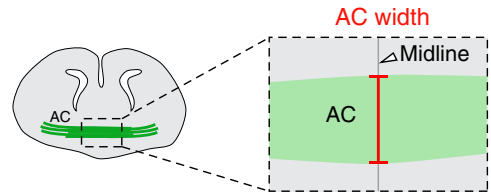

b

Parasagittal
section anti-L1CAM antibody

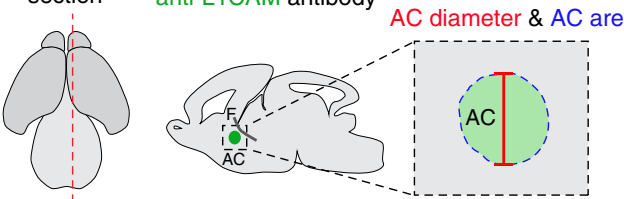

E17.5
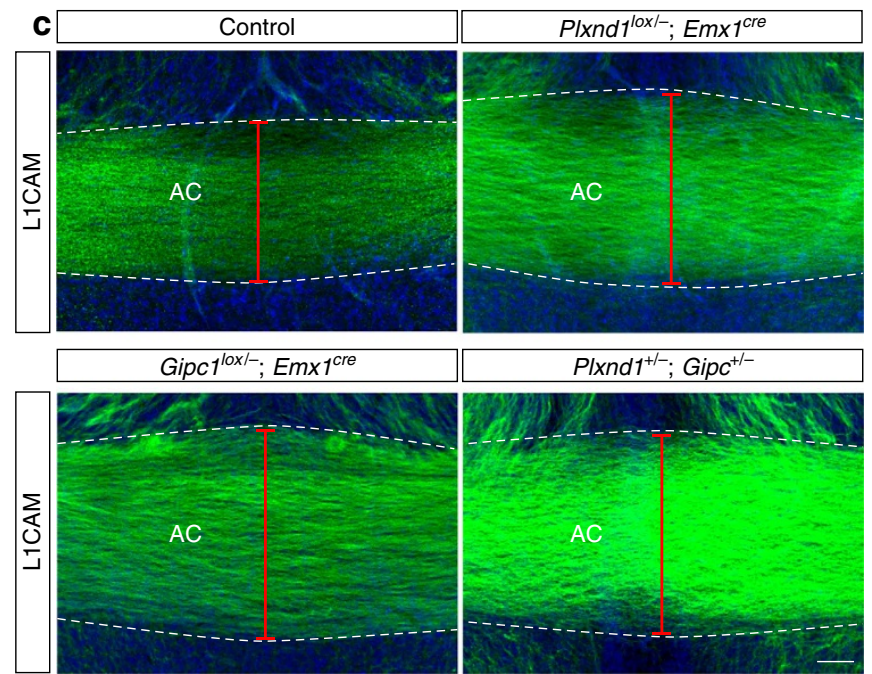

f

e

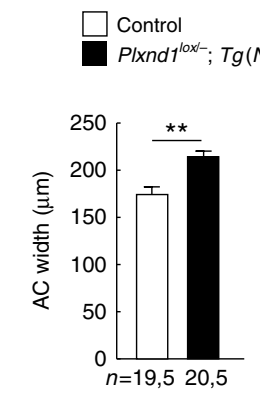

g

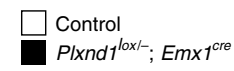

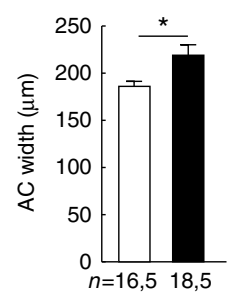

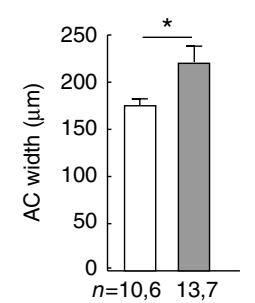

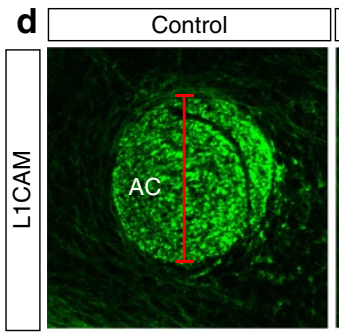
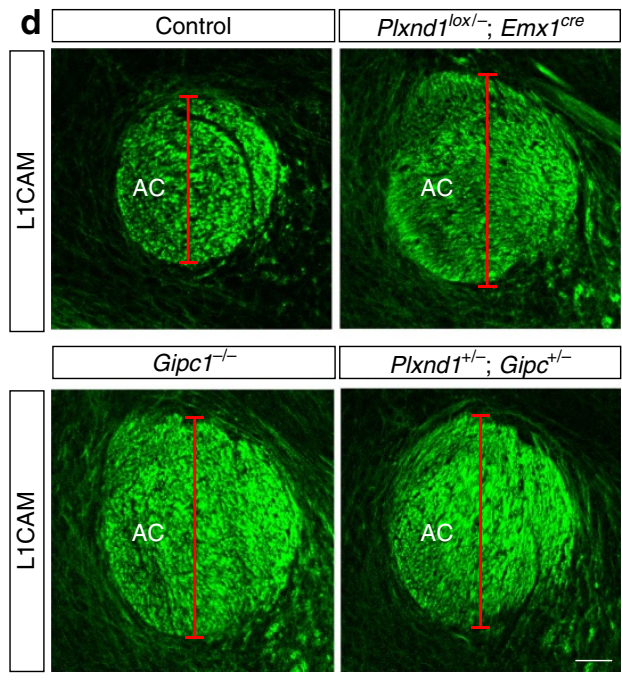

h

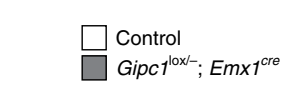

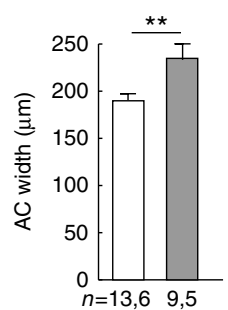
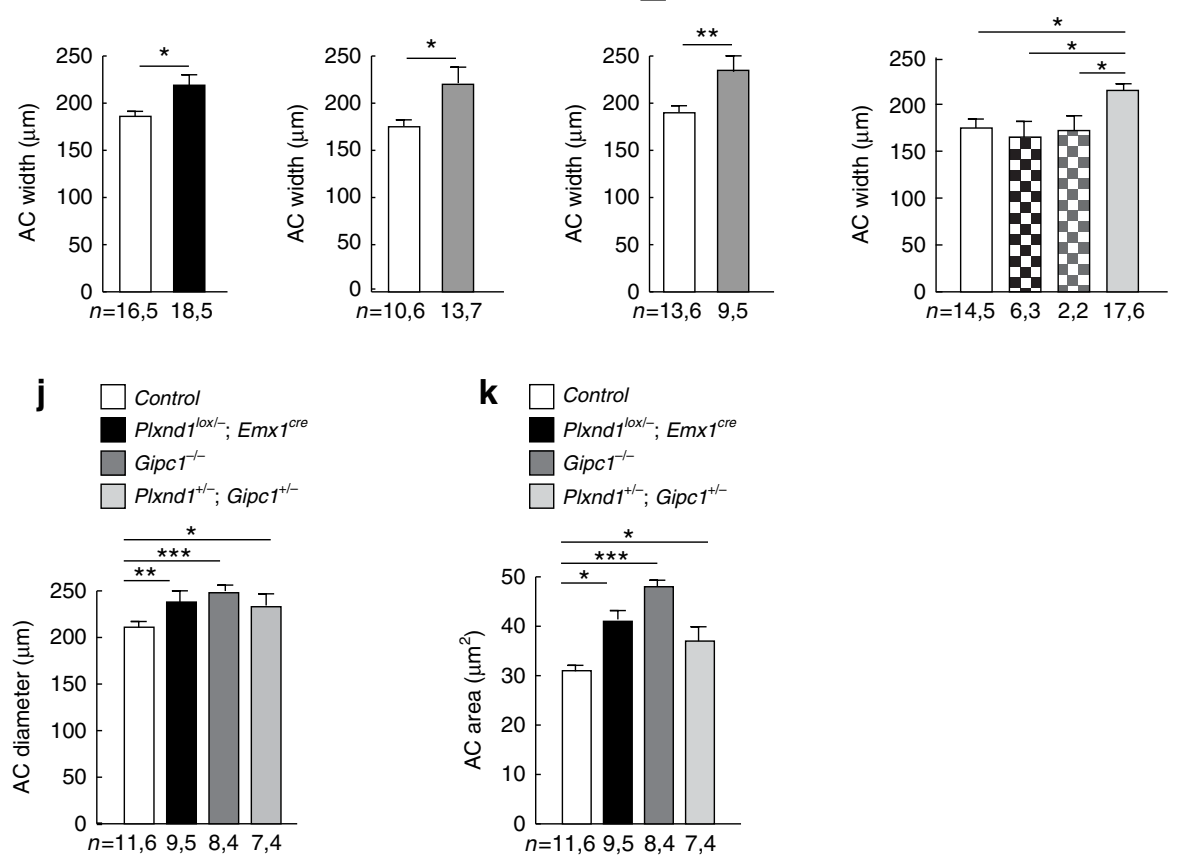

Figure 9 | Plxnd1 and Gipc1 genetically interact to regulate the development of the AC. (a,b) Schematic illustrating the section planes and measurement methods for analysis of the AC. (c,d) Representative L1CAM-stained AC in coronal sections (c) and parasagittal sections at the level of the fornix (F)

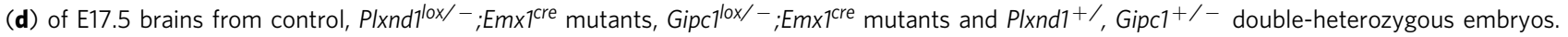

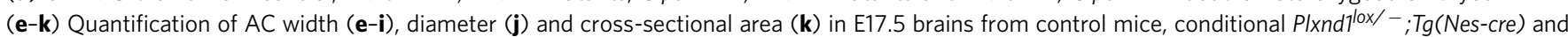

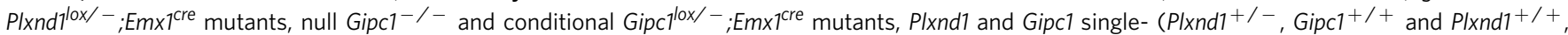
$\mathrm{Gipc1}^{+/-}$) and double-heterozygous (Plxnd1 ${ }^{+/-}, \mathrm{Gipc}^{+/-}$) mutants. Mice lacking Plexin-D1 and/or GIPC1 developed an enlarged AC. Data are shown as mean \pm s.e.m., $n=x, y$ where $x$ indicates the number of slices and $y$ the number of mice analysed for each genotype. ${ }^{\star} P<0.05,{ }^{\star}{ }^{\star} P<0.01$, by the Mann-Whitney test (e-h), Kruskal-Wallis test (i-k). Scale bars, $50 \mu \mathrm{m}$ (c), $40 \mu \mathrm{m}$ (d). See also Supplementary Fig. 7. AC, anterior commissure. F, fornix. 
a

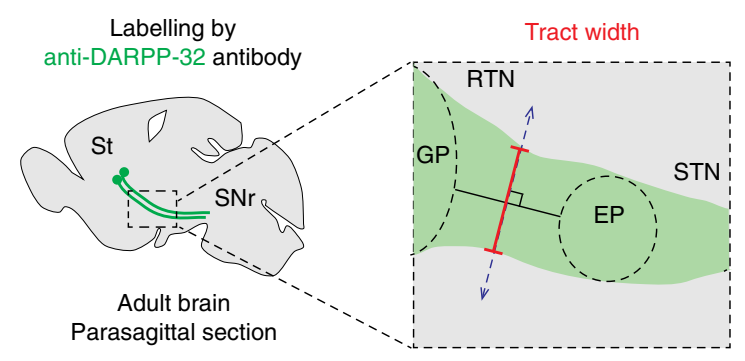

C
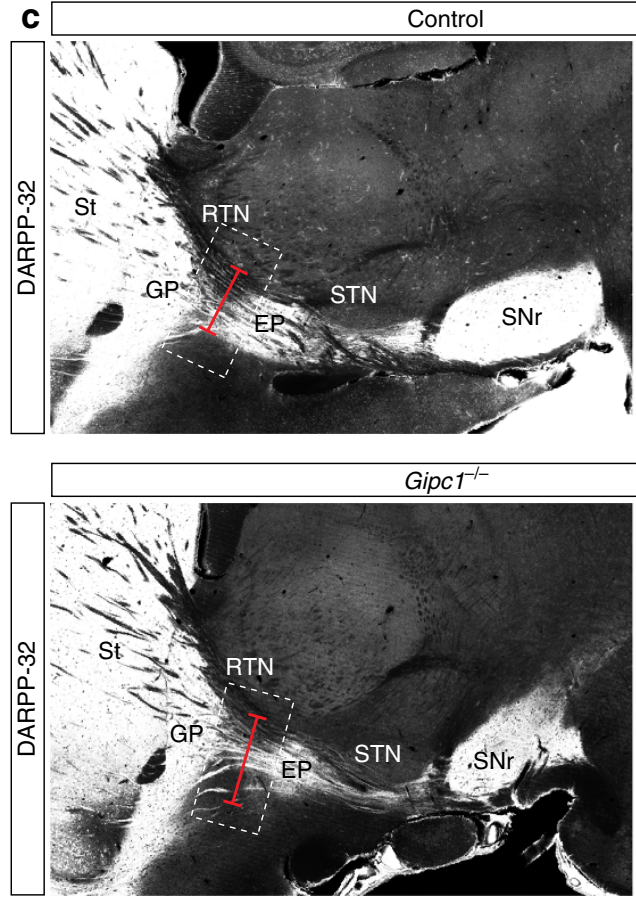

d b
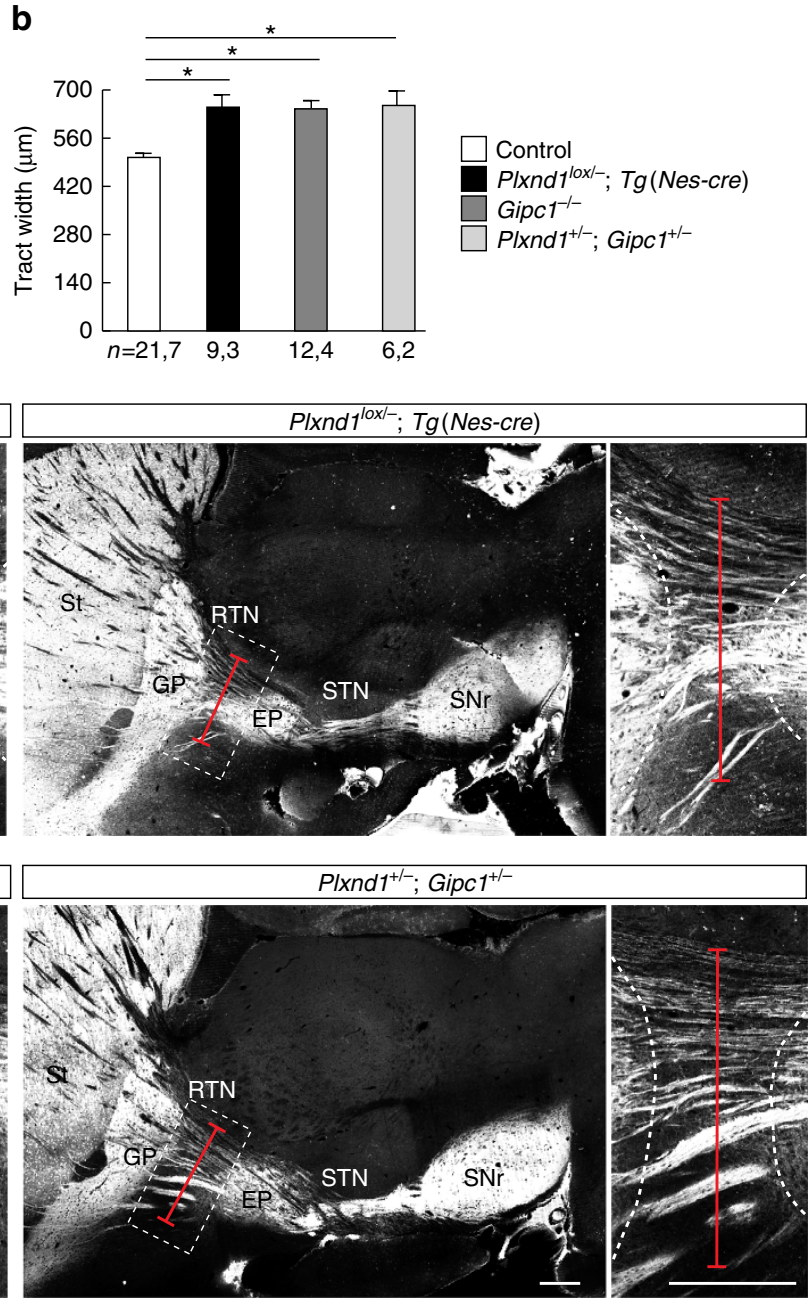

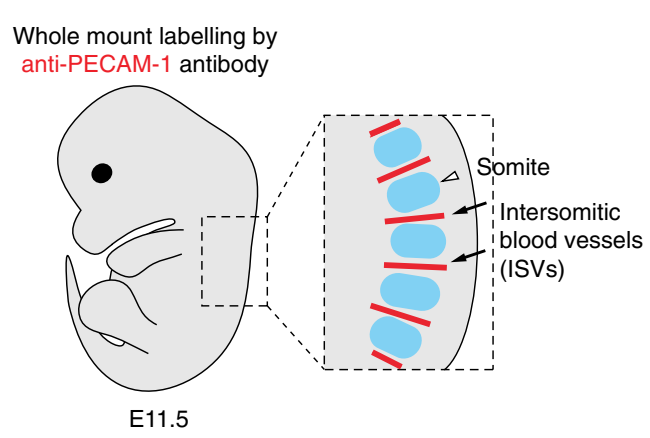

e

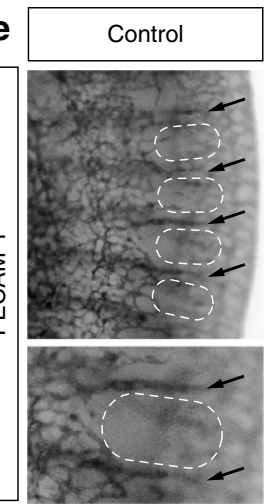

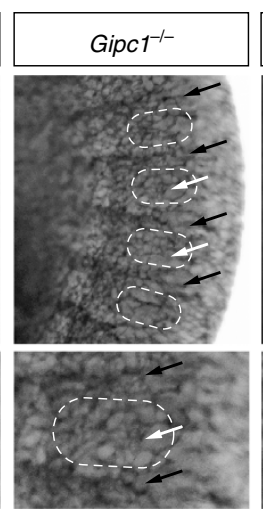

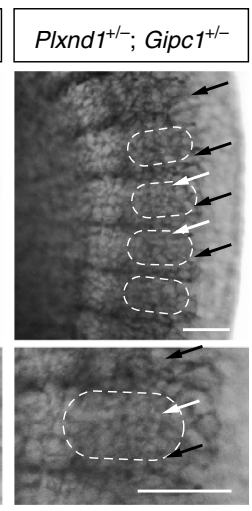

Figure 10 | PIxnd1 and Gipc1 genetically interact to regulate the development of the striatonigral pathway and intersomitic blood vessels.

(a) Schematic representing the region of interest in parasagittal sections of adult mouse brains and the quantification method for analysis of the striatonigral tract. The width (red segment) of the striatonigral tract (green) was measured at equal distance between the border of the globus pallidus (GP) and the entopeduncular nucleus (EP) on parasagittal sections, along a line perpendicular (dashed blue line) to the main orientation of the tract.

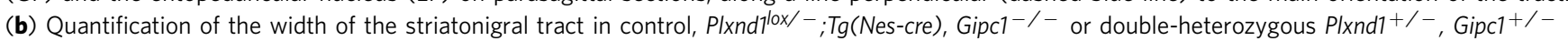
mutant brains. Mice lacking Plexin-D1 or GIPC1 and double heterozygous mice displayed an enlargement of the striatonigral axon tract. Data are shown as mean \pm s.e.m., $n=x, y$ where $x$ indicates the number of slices and $y$ the number of mice analysed for each genotype. ${ }^{\star} P<0.05$, by the Kruskal-Wallis test.

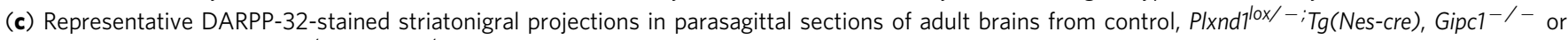
double-heterozygous Plxnd1 ${ }^{+} /-$, Gipc1 ${ }^{+} /-$mutant mice. Red segments delineate the tract width. Onsets show high magnifications views of the tract (dashed boxes in main pictures). (d) Schematic drawing showing the region of interest for analysis of intersomitic blood vessels (ISVs). (e) Whole-mount PECAM-1 staining of E11.5 embryos from control $\left(n=15\right.$ mice), Gipc1 ${ }^{-/-}(n=6$ mice $)$ and double-heterozygous Plxnd1 ${ }^{+/-}$, Gipc $1^{+/-}$mutants $(n=5$ mice). Dashed oval, somite; black arrow, ISV; white arrows, misguided ISV. Mice lacking GIPC1 and double heterozygous mice show disruption of the ISV vascular pattern. EP, entopeduncular nucleus; GP, globus pallidus; ISVs, intersomitic blood vessels; RTN, reticular thalamic nucleus; SNr, substantia nigra; St: striatum; STN, subthalamic nucleus. Scale bars, $500 \mu \mathrm{m}$. See also Supplementary Fig. 8. 
complex, an Arp2/3 activator, promotes the formation of branched actin filaments on endosomal tubules ${ }^{44}$. The molecular motors Myosin VI and Myosin Ic might employ these WASH complex-generated actin filaments to direct cargo proteins to endosomal tubules ${ }^{44}$. Interestingly, GIPC1 possesses a Myosin VI-binding site, and the two proteins have been shown to bind and regulate protein trafficking in a number of cell types ${ }^{45,46}$. However, expressing a dominant negative Myosin VI construct in Pir neurons did not impair axonal response to Sema3E (Supplementary Fig. 9), indicating that GIPC1 functions independently of Myosin VI. Further studies are needed to determine whether GIPC1 contributes to retromer-dependent trafficking events by serving as an adaptor protein linking receptors to components of the retromer, WASH complex and/or to actin motors.

So far, there have been few tests of the significance of endocytic trafficking of guidance receptors in the establishment of neural circuits in vivo. Blocking endocytic removal of guidance receptors from the cell surface (including Eph, TrkA and Robo receptors) has been shown to cause defects in axon pathfinding consistent with defective receptor signalling ${ }^{9,47,48}$. The importance of the intracellular machinery by which neurons degrade or recycle proteins after endocytosis has also been reported ${ }^{49-51}$. However, how inappropriate sorting of internalized guidance receptors to either fate leads to defects in axonal tract formation had not been tested. The present study provides in vivo evidence that the sorting adaptor GIPC1 is required for the development of two major projections from the cortex and striatum in the mouse brain, and plays additional function in guiding blood vessel patterning in the trunk.

In endothelial cells, GIPC1 has been previously reported to interact with the C-terminus SEA motif of the guidance receptor Neuropilin-1 (Nrp1) to modulate responses to semaphorin ligands and VEGF ${ }^{52,53}$. However, this mechanism is unlikely to be involved in the developing ISVs, since mice lacking the intracellular domain of Nrp1, and therefore the GIPC1-binding motif, do not have obvious defects in angiogenesis of ISVs ${ }^{54}$. It is also unlikely that GIPC1 mediates Nrp1-Sema or Nrp1-VEGF signalling in the nervous system because Gipc1-deficient neurons responded normally to the in vitro collapsing activity of several neuropilin-binding semaphorins, including Sema3B and Sema3C. Rather, the neuronal and vascular defects that we observed in Gipc1 mutants likely results from Plexin-D1 receptor misrouting and the consequent loss of repulsive signalling, as further supported by the genetic interaction between Gipc1 and Plxnd1.

Recently, the Drosophila GIPC homologue (dGIPC) has been linked to repulsive semaphorin/plexin signalling during motor axon guidance ${ }^{55}$. In contrast to the present model, dGIPC did not directly interact with the plexin receptor but served to target or stabilize the plexin-associated receptor guanylyl cyclase Gyc76c at the cell surface to promote the semaphorin-induced production of $\mathrm{cGMP}^{55,56}$. The functional consequences of regulated receptor trafficking for the physiological and pathological development of brain connectivity will continue to emerge as the intracellular trafficking routes of more guidance receptors and the mechanisms regulating their post-endocytic sorting are characterized.

\section{Methods}

Outcome assessment. All analyses were performed with the experimenter blind to genotypes and treatment conditions.

Reagents and antibodies. The following reagents were used: mouse Sema3B-Fc (10 nM, R\&D Systems), mouse Sema3C-Fc (10 nM, R\&D Systems), mouse Sema3E-Fc (10 nM, R\&D Systems), Texas Red-X phalloidin (1:50, Life Technologies), calcein-AM (1 $\mu \mathrm{M}$, Sigma-Aldrich), Pitstop 2 and Pitstop 2-negative control $(10 \mu \mathrm{M}, \mathrm{Abcam})$ and dynasore $(80 \mu \mathrm{M}$, Sigma-Aldrich). Antibodies include goat anti-PlexinD1 (1:100, R\&D Systems, Cat. No. AF4160), anti-PlexinD1 (1:200, Abcam, Cat. No. 93234), rat anti-L1CAM (1:500, Millipore, Cat. No. MAB5272), rabbit anti-GIPC1 (1:150, GeneTex, Cat. No. GTX78211), mouse anti$\alpha$-tubulin (1:2,000, Sigma-Aldrich, Cat. No. T9026), rabbit anti-GFP (1:500, Torey Pines Biolabs, Cat. No. TP401), chicken anti-GFP (1:500, Aves Labs, Cat. No. 1020), rabbit anti-TBR1 (1:500, Abcam, Cat. No. 31940), rat anti-CTIP2 (1:250, Abcam, Cat. No. 18465), mouse anti-Myc (1:300, Sigma-Aldrich, Cat. No. M4439), horseradish peroxidase (HRP)-conjugated anti-VSV-G tag antibody (1:3,000, Abcam, Cat. No. 3556), mouse anti-FLAG (1:800, Clone M1, Sigma-Aldrich, Cat. No. F3040), rabbit anti-FLAG (1:4,000, Sigma-Aldrich, Cat. No. F7425), rabbit anti-phospho-S473-Akt (1:1,000, Promega, Cat. No. G744A), rabbit anti-Akt (1:1,000, Cell Signaling, Cat. No. 9272), rat anti-PECAM-1 (1:150, BD Pharmingen, Cat. No. 553370) and goat anti-DARPP32 (1:200, Santa Cruz, Cat. No. 8483). The Alexa Fluor 488 Protein Labelling Kit (Invitrogen) was used to label the mouse anti-FLAG antibody. We used appropriate secondary antibodies that were either conjugated to HRP (Vector Laboratories) or fluorescently labelled (Life Technologies).

Plasmids. Expression constructs encoding VSV-tagged human Plexin-D1 and mouse AP-Sema3E-6xHis (AP-Sema3E) were reported previously ${ }^{16,35}$. The PlexinD1 mutant lacking the SEA motif (VSV-Plexin-D1 $\Delta$ SEA) was generated by PCR-mediated mutagenesis from the VSV-Plexin-D1 expression vector using the following primers: $5^{\prime}$-GTGCTACTAGTAGGCCTGAGACACATGGAGAG TTGGTCAGGC- $3^{\prime}$ and $5^{\prime}$-CAGGCCTACTAGTAGCACTCGTAGATG TTGTCCTCCATCAAAGCC- $3^{\prime}$. The VSV-PlexinB1, VSV-PlexinB2 and VSV-PlexinB3 expression vectors were gifts from A. Püschel ${ }^{57}$. The GFP-Sema3E and FLAG-Plexin-D1 plasmid were generated commercially by GeneCust. The FLAG-GIPC1 construct was a gift from M.G. Farquhar's Lab ${ }^{58}$. The CLC-CFP construct was a gift of R. Jacob ${ }^{59}$. R-Ras ${ }^{38 V}$ was a gift from A. Hall's lab ${ }^{60}$, R-ras Raichu, pCXN2-5MycRab4, pCXN2-5MycRab5a and pCXN2-5MycRab11 were gifts of M. Matsuda ${ }^{26}$ and myrAkt $\Delta 4-129$ was a gift from D. Kaplan ${ }^{61}$.

Animals. All animal procedures were conducted in accordance with the guidelines from the French Ministry of Agriculture (agreement number F1305521) and approved by the local ethics committee (C2EA-14 agreement 2015060510102024 V7 \#1186). Plxnd1 null and conditional Plxnd1;Emx1 $1^{\text {cre }}$ mice have been reported previously $16,62,63$. Plxnd $1^{-1+}$ mice were crossed with $\mathrm{Tg}(\mathrm{Nes}-\mathrm{Cre})$ mice $^{64}$ to generate $P l x n d 1^{-1+} ; \operatorname{Tg}($ Nes-cre $)$ males. Plxnd1 ${ }^{-1+} ; \operatorname{Tg}$ (Nes-cre) (or Plxnd $1^{-/+} ; E m x 1^{\text {cre }}$ ) males were crossed to Plxnd1 ${ }^{\text {lox/lox }}$ (ref. 65) females to generate Plxnd1 ${ }^{\text {lox } /-} ; \mathrm{Tg}(\mathrm{Nes}$-cre $)\left(\right.$ or Plxnd1 ${ }^{\text {lox/ } /} ;$ Emx $\left.1^{\text {cre }}\right)$ mutants and littermate controls, including Plxnd1 ${ }^{l o x /+} ; \mathrm{Tg}\left(\mathrm{Nes}\right.$-cre) (or Plxnd1 $1^{\text {lox } /+} ; E m \times 1^{\text {cre }}$ ), Plxnd1 $1^{+/ l o x}$ and $P l x n d 1^{-/ l o x}$ mice. The control genotypes did not show significant differences and were pooled into a single group for this study. The genotype of the offspring was determined by $\mathrm{PCR}^{16,62}$. Gipc1 ${ }^{-/-}$mice were obtained by crossing the Gipc1 lox/lox (ref. 66) with a ubiquitous deleter cre [B6.C-Tg(CMV-cre) $1 \mathrm{Cgn} / \mathrm{J}]^{67}$ and crossed with $E m \times 1^{\text {cre }}$ to get Gipc1 ${ }^{-/+} ; E m x 1^{\text {cre }}$ males. Gipc1 ${ }^{-/+} ; E m \times 1^{\text {cre }}$ males were then crossed to Gipc1 ${ }^{\text {lox/lox }}$ females to generate Gipc1 ${ }^{\text {lox } /-} ; E m x 1^{\text {cre }}$ mutants and the negative controls Gipc1 lox/+ $; E m x 1^{c r e}$, Gipc1 ${ }^{+/ l o x}$ and Gipc1 ${ }^{-/ l o x}$ that were used in this analysis. The three control genotypes did not show significant differences and were pooled into a single group. The Gipc1 floxed allele was genotyped with the following primer pair: lox-Fwd, $5^{\prime}$-AAGCAAAG GACAGTGCCAGT- $3^{\prime}$ and lox-Rev, 5'-GGACCCACATACCTAGACTGC-3'; the Gipc1 null allele was genotyped with the lox-Fwd primer and null-Rev, $5^{\prime}$-ACAACCTCCGAGCCTCATAA-3'. Transgenic mice expressing GFP under the control of the Plxnd1 promoter [Tg(Plxnd1-EGFP)HF78Gsat/Mmucd] were purchased from the Mutant Mouse Resource Research Centers (MMRRC).

Explant and dissociated neuronal cultures. Embryonic brains from wild-type CD1 mice, Plxnd1 mutants, Gipc1 mutants or control littermates were dissected to extract the Pir cortex at E15.5, the neocortex at E15.5 or the subiculum at E17.5. A detailed protocol for dissociated cultures and electroporation is available in Chauvet et al. ${ }^{6}$.

Growth cone collapse assays. After $48 \mathrm{~h}$ in culture, dissociated E15.5 Pir neurons were incubated with recombinant Sema3B, Sema3C or Sema3E for $20 \mathrm{~min}$ at $37^{\circ} \mathrm{C}$ fixed in $4 \%$ paraformaldehyde (PFA), immunostained with mouse anti-tubulin antibody and labelled with Texas Red-X Phalloidin. Fluorescent-stained growth cones were imaged with a confocal microscope (Zeiss LSM 510 Meta) equipped with a $63 \times$-oil Plan-NEOFLUAR objective. Growth cones were scored as collapsed if their peripheral lamellipodia were absent, and if they had fewer than three filopodia or as non-collapsed. Data were pooled from three independent experiments and the percentages of collapsed and non-collapsed growth cones were calculated for each condition. The statistical significance of differences between conditions was evaluated using the $\chi^{2}$ test.

Axonal growth assays. Dissociated neurons from E17.5 subiculum were cultured for 2-3 days in vitro in the presence or absence of Sema3E, fixed in $4 \%$ PFA and 
immunostained with mouse anti-tubulin antibody or rabbit anti-GFP antibody. Axonal length was quantified using the ImageJ plugin Neuron $J^{16}$. Data were pooled from three independent experiments and mean was calculated. Statistical significance of differences between means was evaluated using the Mann-Whitney test.

Fasciculation assays. Explants of E15.5 Pir cortex grown for $48 \mathrm{~h}$ in the presence or absence of Sema3E were incubated with calcein-AM for $30 \mathrm{~min}$ at $37^{\circ} \mathrm{C}$. Fluorescent-stained explants were imaged with an inversed microscope (Zeiss AxioObserver D1) equipped with a $20 \times$ Plan-NEOFLUAR objective. Quantification of axon fasciculation was performed by measuring fibre bundle thickness at $100 \mu \mathrm{m}$ from the explant's edge. Data were pooled from three independent experiments and mean was calculated. Statistical significance of differences between means was evaluated using the Mann-Whitney test.

\section{Receptor internalization assays. After $48 \mathrm{~h}$ in culture, dissociated E15.5 Pir} neurons expressing VSV-PlexinD1 or VSV-PlexinD1 $\Delta$ SEA were incubated with Sema3B, Sema3C or Sema3E for $10 \mathrm{~min}$ at $37^{\circ} \mathrm{C}$ and fixed in $4 \%$ PFA. Immunostaining with goat anti-Plexin-D1 antibody under non-permeabilizing conditions was performed to detect surface-expressed receptors, and total expression was detected after permeabilization with $0.1 \%$ Triton X-100. Fluorescent-stained growth cones were imaged with a confocal microscope (Zeiss LSM 510 Meta) equipped with a $63 \times$-oil Plan-NEOFLUAR objective. Parameters of time interval and gain setting on the camera were adjusted so that the brightest areas did not reach saturation. The immunofluorescence within the area of the growth cone was measured using ImageJ and the ratio of surface fluorescence to total fluorescence was calculated for each growth cone analysed. Data were pooled from three independent experiments and mean was calculated. Statistical significance of differences between means was evaluated using the Mann-Whitney test.

For antibody-feeding assay, neurons expressing FLAG-Plexin-D1 were surface labelled with Alexa Fluor 488-conjugated anti-FLAG antibody for $15 \mathrm{~min}$ at $37^{\circ} \mathrm{C}$ and were incubated or not with Sema3E for $10 \mathrm{~min}$ at $37^{\circ} \mathrm{C}$. Anti-FLAG antibodies bound to noninternalized receptors were stripped from the cell surface by washing the cells quickly in phosphate-buffered saline lacking $\mathrm{Ca} 2+$ and $\mathrm{Mg} 2+$ supplemented with $0.04 \%$ EDTA, leaving behind only antibody bound to the internalized pool of receptors. Cells were fixed with $4 \%$ formaldehyde and $15 \%$ sucrose for $10 \mathrm{~min}$ at room temperature. Growth cones were imaged with a confocal microscope (Zeiss LSM 510 Meta) equipped with a $63 \times$-oil Plan-NEOFLUAR. Receptor endocytosis was quantified for each growth cone by measuring green fluorescence intensity using ImageJ. The mean fluorescence intensity was calculated and was expressed in arbitrary unit. The statistical significance of differences between means was evaluated using the Mann-Whitney test.

\section{Receptor intracellular trafficking and colocalization assays. After $48 \mathrm{~h}$ in} culture, dissociated Pir neurons transfected with two different expression vectors were incubated with Sema3E for $10 \mathrm{~min}$ at $37^{\circ} \mathrm{C}$. Dual-colour immunostaining with the required primary antibodies were performed under permeabilized condition to detect coexpressed proteins. Growth cones were imaged with a confocal microscope (Zeiss LSM 510 Meta) equipped with a $63 \times$-oil Plan-NEOFLUAR objective. Parameters of time interval and gain setting on the camera were adjusted so that the brightest areas did not reach saturation. For each growth cone, the level of colocalization was measured by calculating the Manders coefficients in ImageJ software using the JACoP plug-in ref. 69. Data were pooled from three independent experiments and mean was calculated. The statistical significance of differences between means was evaluated using the Mann-Whitney test.

Receptor recycling assay. This assay has been described in Choy et al. ${ }^{23}$. Briefly, neurons expressing FLAG-Plexin-D1 were surface labelled with Alexa Fluor 488-conjugated anti-FLAG antibody for $15 \mathrm{~min}$ at $37^{\circ} \mathrm{C}$, and were subjected to 3 sets of conditions in parallel as indicated: Condition 1, nontreated (C) - cells were fixed after $30 \mathrm{~min}$ of incubation in the absence of Sema3E and without a surface stripping step; Condition 2, surface stripped (Z) - cells were incubated with Sema3E for $10 \mathrm{~min}$ at $37^{\circ} \mathrm{C}$, followed by a EDTA stripping step to remove residual Alexa Fluor 488-conjugated anti-FLAG antibody from the cell surface by washing the cells quickly in phosphate-buffered saline lacking $\mathrm{Ca} 2+$ and $\mathrm{Mg} 2+$ supplemented with $0.04 \%$ EDTA, leaving behind only antibody bound to the internalized pool of receptors; and Condition 3, surface recovery (E)-EDTAstripped cells as mentioned above were incubated for $45 \mathrm{~min}$ at $37^{\circ} \mathrm{C}$ in fresh Neurobasal medium to let recycling occurred. For all 3 sets of conditions, cells were fixed with $4 \%$ formaldehyde and $15 \%$ sucrose for $10 \mathrm{~min}$ at room temperature under nonpermeabilizing condition, and stained with Alexa Fluor 568-conjugated anti-mouse secondary antibodies to label cell surface FLAG-Plexin-D1 receptors. Growth cones were imaged with a confocal microscope (Zeiss LSM 510 Meta) equipped with a $63 \times$-oil Plan-NEOFLUAR. Ratiometric image analysis was done using ImageJ by calculating the ratio of fluorescence intensity of nonpermeabilizing staining of cell surface FLAG-Plexin-D1 by a secondary antibody (red channel) to the overall intensity of FLAG-Plexin-D1 initially labelled with anti-FLAG antibody on the plasma membrane (green channel). The percentage of receptors recycled in individual cells was calculated from the red/green ratios from the 3 sets of conditions using the following formula: \% Recycling $=(\mathrm{E}-\mathrm{Z}) /(\mathrm{C}-\mathrm{Z}) \times 100 \%$. The average of percentage of recycling was calculated and statistical significance of differences between groups was evaluated using the Mann-Whitney test.

Immunoprecipitation and western blotting. HEK293T cells (ATCC, mycoplasma free tested) were transfected with Flag-GIPC1 and/or equal amounts of VSV-PlexinD1, VSV-PlexinD1DSEA, VSV-PlexinB1, VSV-PlexinB2 or VSV-PlexinB3 expression vectors using Lipofectamine Plus (Invitrogen). At 2 days after transfection, the cell lysates were immunoprecipitated using ANTI-FLAG M2 Affinity Gel (Sigma-Aldrich) or VSV-G tag antibody Agarose. E15.5 Pir cortex cell lysate was immunoprecipitated with anti-GIPC1 antibody immobilized on protein A-Sepharose beads. Akt phosphorylation was evaluated on lysates of E15.5 Pir neurons cultured for 2 days and serum-starved for $2 \mathrm{~h}$ before Sema3E stimulation (from 0 to $30 \mathrm{~min}$ ). Complexes and/or cell lysates were separated by electrophoresis and electrotransferred onto membranes (Immobilon-P, Millipore) Membranes were incubated with HRP-conjugated VSV-G tag, rabbit anti-FLAG, rabbit anti-GIPC1, rabbit anti-PlexinD1, anti-pS473 Akt or anti-Akt antibodies. After incubation with HRP-conjugated secondary antibodies, signals were detected with an enhanced chemiluminescence system (GE Healthcare). Akt activation was quantified by measuring the intensity of hybridized bands using ImageJ software and by calculating the phospho-Akt/Akt intensity ratio for each individual experiment. Ratios obtained from three independent experiments were averaged and statistical difference between means was evaluated by Mann-Whitney test. Images have been cropped for presentation. Full size images are presented in Supplementary Figs 4 and 6 .

Production of fusion proteins and binding assay. Conditioning media containing recombinant mouse AP-Sema3E and GFP-Sema3E proteins were obtained from transiently transfected HEK293T cells ${ }^{68}$. Quantification of GFP-Sema3E was performed using the GFP quantification kit (Biovision). AP-Sema3E binding experiments on COS7 cells (ATCC) were performed by conventional methods ${ }^{16}$. For binding experiments on neurons, after $10 \mathrm{~min}$ of treatment with $10 \mathrm{nM}$ GFP-Sema3E, dissociated neurons were fixed in 4\% PFA and immunostained with chicken anti-GFP antibody. The mean intensity of fluorescence per pixel was measured using ImageJ software and multiplied by the surface area of the growth cone to evaluate the binding of GFP-Sema3E per growth cone. Data were pooled from three independent experiments and mean was measured. The statistical significance of differences between the groups was evaluated using the Mann-Whitney test.

FRET experiments. Dissociated E15.5 Pir neurons of wild-type CD1 or Gipc1 ${ }^{-/-}$ mutant embryos were electroporated with the FRET biosensor Raichu R-ras and plated on glass-bottom dishes (MatTek). After 2 days in culture, neurons were imaged in a controlled atmosphere on the heated stage $\left(37^{\circ} \mathrm{C}\right)$ of an inverted spinning disk Eclipse TI microscope (Nikon) controlled by MetaMorph. Images for cyan fluorescent protein (CFP; excitation $445 \mathrm{~nm}$, emission $495 \mathrm{~nm}$ ) and yellow fluorescent protein (YFP; excitation $445 \mathrm{~nm}$, emission $515 \mathrm{~nm}$ ) were acquired with a 2 EMCCD camera (Photometrics) every $20 \mathrm{~s}$ from $6 \mathrm{~min}$ before to $12 \mathrm{~min}$ after the addition of Sema3E to the medium. YFP/CFP ratio images were generated with ImageJ software to represent the levels of FRET used for quantitative analyses. Mean ratios of randomly selected ROIs defined by individual vesicles were obtained for a period of $6 \mathrm{~min}(0-6 \mathrm{~min}$, control) before Sema3E and were compared with the $6 \mathrm{~min}$ period (3-9 $\mathrm{min}$ ) following Sema3E application. Changes of $>10 \%$ of the mean control value were considered increases or decreases. Data are presented as percentage of vesicles showing increased, decreased or unchanged FRET levels. The statistical significance of differences between the groups was evaluated using the $\chi^{2}$ test.

Immunohistochemistry. Brains were sectioned, at thickness of $80-100 \mu \mathrm{m}$, on a vibratome and immunohistochemistry on floating sections was performed following common procedures. Fluorescent-stained brain sections were imaged with an AxioImager Z1 Apotome controlled by Axiovision Imagery or with a confocal LSM 780 controlled by zen2010 software (Zeiss). The dorsoventral width of the AC was measured using ImageJ software at the level of the midline on all coronal sections through the body of the AC ( $2-3$ sections per brain). The diameter and cross-sectional area of the $\mathrm{AC}$ were measured using ImageJ software on parasagittal sections taken at comparable mediolateral levels using the fornix as a landmark. The striatonigral tract was analysed on parasagittal sections in which the entire pathway could be seen from the globus pallidus to the substantia nigra (three sections per hemi-brain). The width of the tract was measured at mid-distance between the external border of the globus pallidus and the entopeduncular nucleus (visualized by DAPI staining), along an axis placed orthogonally to the orientation of the tract. For all analyses, 2-7 animals from at least 2 different litters were analysed for each genotype. The mean of measurements was calculated and statistical significance of differences between means was evaluated using the Mann-Whitney or the Kruskal-Wallis test. 
For CTIP2/TRBR1 colocalization analyses, 2-3 animals from at least 2 different litters were analysed for each genotype. The percentage of co-labelled cells was calculated and statistical significance of differences between the groups was evaluated using the $\chi^{2}$ test.

Immunohistochemistry on whole-mount embryos with antibody against PECAM-1 was performed following standard procedures. Embryos were imaged with a Zeiss stereomicroscope Lumar. For each genotype, 5-15 animals from 3 different litters were analysed.

In situ hybridization. In situ hybridization was performed on $100-\mu \mathrm{m}$-thick vibratome sections of E15.5 and E17.5 mouse brains following standard protocols. Plxnd1 and Sema3e probes were generously provided by M. Tessier-Lavigne and C. Christensen ${ }^{70}$, respectively. The sequence 1,083 to 1,576 of the $3^{\prime}$ untranslated region of Gipc1 cDNA (NM-018771.3) was cloned into pCR Topo Blunt II to obtain a Gipcl probe template. The vector was linearized by digestion with EcoRV, and antisense RNA probe was synthetized by Sp6 polymerase. The same vector was linearized by BamHI and transcribed by T7 polymerase to obtain a sense RNA probe as a control.

Axonal tracing. For axonal tracing, embryonic brains were fixed at least overnight in $4 \%$ PFA at $4{ }^{\circ} \mathrm{C}$. Small crystals of DiI $\left(1,1^{\prime}\right.$-dioctadecyl 3,3,3, $3^{\prime}$-tetramethylindocarbo-cyanine perchlorate; Molecular Probes) were inserted into the cerebral cortex and allowed to diffuse at $37^{\circ} \mathrm{C}$ for 2 weeks. Brains were cut into $100-\mu \mathrm{m}$-thick vibratome sections, and tracing specificity was systematically confirmed after diffusion on serial sections adjacent to the site of crystal insertion. Fluorescent-stained brain sections were imaged with an AxioImager Z1 Apotome controlled by Axiovision Imagery.

Statistics. For each experiment, the normal distribution of the data was examined using a D'Agostino-Pearson omnibus test for sample sizes of 6 or higher. The estimate of variance was determined by the s.d. of each group that was similar between groups. Since data were nonparametric, Mann-Whitney test was used to compare two group means and Kruskal-Wallis test to compare differences between more than two groups. When data were distributed across categories, we used the $\chi^{2}$ test. All analyses were performed using the Prism6 software. Statistical significance was set at $P<0.05$. No statistical methods were used to predetermine sample sizes, but our sample sizes are similar to those generally employed in the field.

Data availability. All relevant data are available from the authors.

\section{References}

1. Itofusa, R. \& Kamiguchi, H. Polarizing membrane dynamics and adhesion for growth cone navigation. Mol. Cell. Neurosci. 48, 332-338 (2011).

2. Pfenninger, K. H. Plasma membrane expansion: a neuron's Herculean task. Nat. Rev. Neurosci. 10, 251-261 (2009).

3. Zylbersztejn, K. \& Galli, T. [Membrane traffic, a new actor in axon guidance]. Med. Sci. 28, 267-269 (2012).

4. Tojima, T., Hines, J. H., Henley, J. R. \& Kamiguchi, H. Second messengers and membrane trafficking direct and organize growth cone steering. Nat. Rev. Neurosci. 12, 191-203 (2011).

5. Tojima, T. \& Kamiguchi, H. Exocytic and endocytic membrane trafficking in axon development. Dev. Growth Differ. 57, 291-304 (2015).

6. Winckler, B. \& Mellman, I. Trafficking guidance receptors. Cold Spring Harb. Perspect. Biol. 2, a001826 (2010)

7. Piper, M., Salih, S., Weinl, C., Holt, C. E. \& Harris, W. A. Endocytosisdependent desensitization and protein synthesis-dependent resensitization in retinal growth cone adaptation. Nat. Neurosci. 8, 179-186 (2005).

8. Shafer, B., Onishi, K., Lo, C., Colakoglu, G. \& Zou, Y. Vangl2 promotes Wnt/planar cell polarity-like signaling by antagonizing Dvll-mediated feedback inhibition in growth cone guidance. Dev. Cell. 20, 177-191 (2011).

9. Chance, R. K. \& Bashaw, G. J. Slit-dependent endocytic trafficking of the robo receptor is required for son of sevenless recruitment and midline axon repulsion. PLoS Genet. 11, e1005402 (2015).

10. Irannejad, R., Tsvetanova, N. G., Lobingier, B. T. \& von Zastrow, M. Effects of endocytosis on receptor-mediated signaling. Curr. Opin. Cell Biol. 35, 137-143 (2015).

11. Fournier, A. E. et al. Semaphorin3A enhances endocytosis at sites of receptorF-actin colocalization during growth cone collapse. J. Cell Biol. 149, 411-422 (2000).

12. Dang, P., Smythe, E. \& Furley, A. J. TAG1 regulates the endocytic trafficking and signaling of the semaphorin3A receptor complex. J. Neurosc. 32, 10370-10382 (2012)

13. Law, C. O., Kirby, R. J., Aghamohammadzadeh, S. \& Furley, A. J. The neural adhesion molecule TAG-1 modulates responses of sensory axons to diffusible guidance signals. Development 135, 2361-2371 (2008).
14. Gu, C. et al. Semaphorin $3 \mathrm{E}$ and plexin-D1 control vascular pattern independently of neuropilins. Science 307, 265-268 (2005).

15. Oh, W. J. \& Gu, C. The role and mechanism-of-action of Sema3E and PlexinD1 in vascular and neural development. Semin. Cell Dev. Biol. 24, 156-162 (2013).

16. Chauvet, S. et al. Gating of Sema3E/PlexinD1 signaling by neuropilin-1 switches axonal repulsion to attraction during brain development. Neuron 56, 807-822 (2007).

17. von Kleist, L. et al. Role of the clathrin terminal domain in regulating coated pit dynamics revealed by small molecule inhibition. Cell 146, 471-484 (2011).

18. Macia, E. et al. Dynasore, a cell-permeable inhibitor of dynamin. Dev. Cell 10, 839-850 (2006).

19. Romero, G., von Zastrow, M. \& Friedman, P. A. Role of PDZ proteins in regulating trafficking, signaling, and function of GPCRs: means, motif, and opportunity. Adv. Pharmacol. 62, 279-314 (2011).

20. Katoh, M. Functional proteomics, human genetics and cancer biology of GIPC family members. Exp. Mol. Med. 45, e26 (2013).

21. Aurandt, J., Vikis, H. G., Gutkind, J. S., Ahn, N. \& Guan, K. L. The semaphorin receptor plexin-B1 signals through a direct interaction with the Rho-specific nucleotide exchange factor, LARG. Proc. Natl Acad. Sci. USA 99, 12085-12090 (2002).

22. Perrot, V., Vazquez-Prado, J. \& Gutkind, J. S. Plexin B regulates Rho through the guanine nucleotide exchange factors leukemia-associated Rho GEF (LARG) and PDZ-RhoGEF. J. Biol. Chem. 277, 43115-43120 (2002).

23. Choy, R. W. et al. Retromer mediates a discrete route of local membrane delivery to dendrites. Neuron 82, 55-62 (2014).

24. Sakurai, A. et al. Semaphorin 3E initiates antiangiogenic signaling through plexin D1 by regulating Arf6 and R-Ras. Mol. Cell. Biol. 30, 3086-3098 (2010).

25. Uesugi, K., Oinuma, I., Katoh, H. \& Negishi, M. Different requirement for Rnd GTPases of R-Ras GAP activity of Plexin-C1 and Plexin-D1. J. Biol. Chem. 284, 6743-6751 (2009).

26. Takaya, A. et al. R-Ras regulates exocytosis by Rgl2/Rlf-mediated activation of RalA on endosomes. Mol. Biol. Cell 18, 1850-1860 (2007).

27. Ivins, J. K., Yurchenco, P. D. \& Lander, A. D. Regulation of neurite outgrowth by integrin activation. J. Neurosci. 20, 6551-6560 (2000).

28. Silver, L., Michael, J. V., Goldfinger, L. E. \& Gallo, G. Activation of PI3K and R-Ras signaling promotes the extension of sensory axons on inhibitory chondroitin sulfate proteoglycans. Dev. Neurobiol. 74, 918-933 (2014).

29. Marte, B. M., Rodriguez-Viciana, P., Wennstrom, S., Warne, P. H. \& Downward, J. R-Ras can activate the phosphoinositide 3-kinase but not the MAP kinase arm of the Ras effector pathways. Curr. Biol. 7, 63-70 (1997).

30. Jouandet, M. L. \& Hartenstein, V. Basal telencephalic origins of the anterior commissure of the rat. Exp. Brain Res. 50, 183-192 (1983).

31. Livy, D. J. et al. Increased axon number in the anterior commissure of mice lacking a corpus callosum. Exp. Neurol. 146, 491-501 (1997).

32. Barr, M. S. \& Corballis, M. C. The role of the anterior commissure in callosal agenesis. Neuropsychology 16, 459-471 (2002).

33. Hannay, H. J., Dennis, M., Kramer, L., Blaser, S. \& Fletcher, J. M. Partial agenesis of the corpus callosum in spina bifida meningomyelocele and potential compensatory mechanisms. J. Clin. Exp. Neuropsychol. 31, 180-194 (2009).

34. Ehrman, L. A. et al. The LIM homeobox gene Isl1 is required for the correct development of the striatonigral pathway in the mouse. Proc. Natl Acad. Sci. USA 110, E4026-E4035 (2013).

35. Bellon, A. et al. VEGFR2 (KDR/Flk1) signaling mediates axon growth in response to semaphorin $3 \mathrm{E}$ in the developing brain. Neuron 66, 205-219 (2010).

36. Wu, K. Y. et al. Semaphorin $3 \mathrm{~A}$ activates the guanosine triphosphatase Rab5 to promote growth cone collapse and organize callosal axon projections. Sci. Signal 7, ra81 (2014)

37. Oinuma, I., Ishikawa, Y., Katoh, H. \& Negishi, M. The Semaphorin 4 D receptor Plexin-B1 is a GTPase activating protein for R-Ras. Science 305, 862-865 (2004).

38. Saito, Y., Oinuma, I., Fujimoto, S. \& Negishi, M. Plexin-B1 is a GTPase activating protein for M-Ras, remodelling dendrite morphology. EMBO Rep. 10, 614-621 (2009)

39. Wang, Y. et al. Plexins are GTPase-activating proteins for Rap and are activated by induced dimerization. Sci. Signal 5, ra6 (2012).

40. Hisata, S. et al. Rap1-PDZ-GEF1 interacts with a neurotrophin receptor at late endosomes, leading to sustained activation of Rap1 and ERK and neurite outgrowth. J. Cell Biol. 178, 843-860 (2007).

41. Pizon, V., Desjardins, M., Bucci, C., Parton, R. G. \& Zerial, M. Association of Rapla and Raplb proteins with late endocytic/phagocytic compartments and Rap2a with the Golgi complex. J. Cell Sci. 107(Pt 6): 1661-1670 (1994).

42. Gallon, M. \& Cullen, P. J. Retromer and sorting nexins in endosomal sorting. Biochem. Soc. Trans. 43, 33-47 (2015).

43. Steinberg, F. et al. A global analysis of SNX27-retromer assembly and cargo specificity reveals a function in glucose and metal ion transport. Nat. Cell Biol. 15, 461-471 (2013). 
44. Seaman, M. N., Gautreau, A. \& Billadeau, D. D. Retromer-mediated endosomal protein sorting: all WASHed up! Trends Cell Biol. 23, 522-528 (2013).

45. Bunn, R. C., Jensen, M. A. \& Reed, B. C. Protein interactions with the glucose transporter binding protein GLUT1CBP that provide a link between GLUT1 and the cytoskeleton. Mol. Biol. Cell 10, 819-832 (1999).

46. Ewing, R. M. et al. Large-scale mapping of human protein-protein interactions by mass spectrometry. Mol. Syst. Biol. 3, 89 (2007).

47. Bodmer, D., Ascano, M. \& Kuruvilla, R. Isoform-specific dephosphorylation of dynamin 1 by calcineurin couples neurotrophin receptor endocytosis to axonal growth. Neuron 70, 1085-1099 (2011).

48. Cowan, C. W. et al. Vav family GEFs link activated Ephs to endocytosis and axon guidance. Neuron 46, 205-217 (2005).

49. Bhuin, T. \& Roy, J. K. Rab11 is required for embryonic nervous system development in Drosophila. Cell Tissue Res. 335, 349-356 (2009).

50. Falk, J., Konopacki, F. A., Zivraj, K. H. \& Holt, C. E. Rab5 and Rab4 regulate axon elongation in the Xenopus visual system. J. Neurosci. 34, 373-391 (2014).

51. Williamson, W. R., Yang, T., Terman, J. R. \& Hiesinger, P. R. Guidance receptor degradation is required for neuronal connectivity in the Drosophila nervous system. PLoS Biol. 8, e1000553 (2010).

52. Salikhova, A. et al. Vascular endothelial growth factor and semaphorin induce neuropilin-1 endocytosis via separate pathways. Circ. Res. 103, e71-e79 (2008).

53. Lanahan, A. et al. The neuropilin 1 cytoplasmic domain is required for VEGFA-dependent arteriogenesis. Dev. Cell 25, 156-168 (2013).

54. Fantin, A. et al. The cytoplasmic domain of neuropilin 1 is dispensable for angiogenesis, but promotes the spatial separation of retinal arteries and veins. Development 138, 4185-4191 (2011).

55. Chak, K. \& Kolodkin, A. L. Function of the Drosophila receptor guanylyl cyclase Gyc76C in PlexA-mediated motor axon guidance. Development 141, 136-147 (2014).

56. Ayoob, J. C., Yu, H. H., Terman, J. R. \& Kolodkin, A. L. The Drosophila receptor guanylyl cyclase Gyc76C is required for semaphorin-1a-plexin A-mediated axonal repulsion. J. Neurosci. 24, 6639-6649 (2004).

57. Rohm, B., Ottemeyer, A., Lohrum, M. \& Puschel, A. W. Plexin/neuropilin complexes mediate repulsion by the axonal guidance signal semaphorin $3 \mathrm{~A}$. Mech. Dev. 93, 95-104 (2000).

58. Varsano, T. et al. GIPC is recruited by APPL to peripheral TrkA endosomes and regulates TrkA trafficking and signaling. Mol. Cell. Biol. 26, 8942-8952 (2006).

59. Straube, T. et al. pH-dependent recycling of galectin-3 at the apical membrane of epithelial cells. Traffic 14, 1014-1027 (2013).

60. Self, A. J., Caron, E., Paterson, H. F. \& Hall, A. Analysis of R-Ras signalling pathways. J. Cell Sci. 114, 1357-1366 (2001).

61. Kohn, A. D., Summers, S. A., Birnbaum, M. J. \& Roth, R. A. Expression of a constitutively active Akt Ser/Thr kinase in 3T3-L1 adipocytes stimulates glucose uptake and glucose transporter 4 translocation. J. Biol. Chem. 271, 31372-31378 (1996).

62. Deck, M. et al. Pathfinding of corticothalamic axons relies on a rendezvous with thalamic projections. Neuron 77, 472-484 (2013).

63. Gorski, J. A. et al. Cortical excitatory neurons and glia, but not GABAergic neurons, are produced in the Emxl-expressing lineage. J. Neurosci. 22, 6309-6314 (2002)

64. Tronche, F. et al. Disruption of the glucocorticoid receptor gene in the nervous system results in reduced anxiety. Nat. Genet. 23, 99-103 (1999).

65. Zhang, Y. et al. Tie2Cre-mediated inactivation of plexinD1 results in congenital heart, vascular and skeletal defects. Dev. Biol. 325, 82-93 (2009).

66. Moraes, F. et al. Endothelial cell-dependent regulation of arteriogenesis. Circ Res. 113, 1076-1086 (2013).
67. Schwenk, F., Baron, U. \& Rajewsky, K. A cre-transgenic mouse strain for the ubiquitous deletion of loxP-flanked gene segments including deletion in germ cells. Nucleic Acids Res. 23, 5080-5081 (1995).

68. Chauvet, S., Mire, E. \& Mann, F. Characterizing Semaphorin signaling using isolated neurons in culture. Methods Mol. Biol. 1493, 223-235 (2017).

69. Bolte, S. \& Cordelieres, F. P. A guided tour into subcellular colocalization analysis in light microscopy. J. Microsc. 224, 213-232 (2006).

70. Christensen, C. R. et al. Transcription of a novel mouse semaphorin gene, $\mathrm{M}$-semaH, correlates with the metastatic ability of mouse tumor cell lines. Cancer Res. 58, 1238-1244 (1998).

\section{Acknowledgements}

We thank Stéphane Nicolas and Laura Miranda for excellent technical assistance and Axelle Dovonou for substantial help with the experiments. We acknowledge the dedicated staff of the IBDM animal house facility and the France-BioImaging infrastructure supported by the Agence Nationale de la Recherche (ANR-10-INSB-04-01, 'Investissements d'Avenir'). This work was supported by the Centre National de la Recherche Scientifique (CNRS), Aix Marseille Université and by grants from the Agence Nationale de la Recherche (ANR-10-BLAN-1412, ANR-12-BSV4-0012-01) to F.Ma., Fédération pour la Recherche sur le Cerveau to F.Ma., Fondation ARC to F.Ma., Institut National du Cancer (2011-139) to F.Ma., Fondation pour la Recherche Médicale (Equipe FRM DEQ20150331728) to F. Ma. and Institut Universitaire de France to S.C.

\section{Author contributions}

Conceptualization: A.B., S.C. and F.Ma. Investigations: K.B. contributed to Figs 1a,c,d, 4a, 6c-g, 7i-l, 8a and 9c,e-i, and Supplementary Figs 3a,j, 4a,b, 5c,d, 6, 7 and $8 \mathrm{~b}$. E.M. contributed to Figs $7 \mathrm{e}-\mathrm{g}, 8 \mathrm{~b}-\mathrm{d}, 9 \mathrm{a}-\mathrm{d}, \mathrm{j}, \mathrm{k}$ and $10 \mathrm{a}-\mathrm{c}$ and Supplementary Fig $3 \mathrm{k}$. A.B. contributed to Figs 1c, 4a-d and 9c,e-i, and Supplementary Figs 3b-d, 3j, 4a,c, 8a,b $8 \mathrm{e}-\mathrm{g}$. M.H. contributed to Figs $8 \mathrm{~b}-\mathrm{e}$ and $9 \mathrm{c}, \mathrm{e}-\mathrm{i}$ and Supplementary Figs $3 \mathrm{~b}$ and $8 \mathrm{~b}$. J.G. contributed to Figs 2f,g, 3e, 4g,h and 5, and Supplementary Figs 2a,b and 3l,m. S.C. contributed to Figs $1 \mathrm{~b}-\mathrm{f}, 2,3,4 \mathrm{c}-\mathrm{l}, 5,6 \mathrm{a}-\mathrm{c}, 7 \mathrm{a}-\mathrm{d}, \mathrm{h}$ and $10 \mathrm{~b}-\mathrm{e}$, and Supplementary Figs 1, 2, 3e-i,l,m, 5a,b, 8c-g and 9. M.S., F.Mo. and Y.Y. provided transgenic adult mice and embryos. Supervision and writing: S.C. and F.Ma.

\section{Additional information}

Supplementary Information accompanies this paper at http://www.nature.com/ naturecommunications

Competing financial interests: The authors declare no competing financial interests.

Reprints and permission information is available online at http://npg.nature.com/ reprintsandpermissions/

How to cite this article: Burk, K. et al. Post-endocytic sorting of Plexin-D1 controls signal transduction and development of axonal and vascular circuits. Nat. Commun. 8, 14508 doi: 10.1038/ncomms14508 (2017).

Publisher's note: Springer Nature remains neutral with regard to jurisdictional claims in published maps and institutional affiliations.

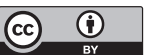

This work is licensed under a Creative Commons Attribution 4.0 International License. The images or other third party material in this article are included in the article's Creative Commons license, unless indicated otherwise in the credit line; if the material is not included under the Creative Commons license, users will need to obtain permission from the license holder to reproduce the material. To view a copy of this license, visit http://creativecommons.org/licenses/by/4.0/

C) The Author(s) 2017 